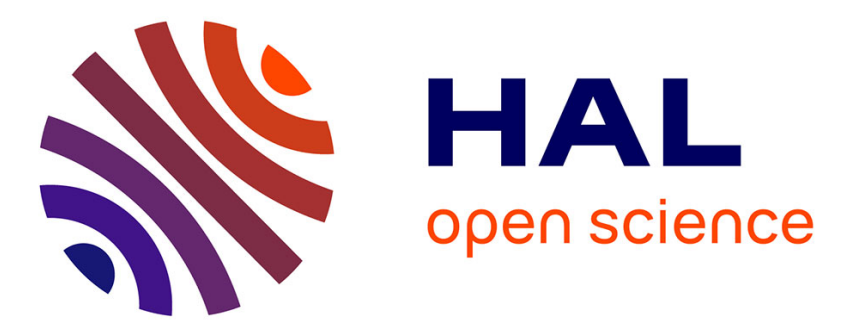

\title{
Exploitation du cheval au Magdalénien supérieur dans le Sud-Ouest de la France: Le cas de l'abri Faustin (Cessac, Gironde)
}

\author{
Léa Feyfant, David Cochard, Jean-Baptiste Mallye
}

\section{To cite this version: \\ Léa Feyfant, David Cochard, Jean-Baptiste Mallye. Exploitation du cheval au Magdalénien supérieur dans le Sud-Ouest de la France: Le cas de l'abri Faustin (Cessac, Gironde). Bulletin de la Société préhistorique française, 2015, 112 (4), pp.693-716. hal-01958876}

\section{HAL Id: hal-01958876 https://hal.science/hal-01958876}

Submitted on 18 Dec 2018

HAL is a multi-disciplinary open access archive for the deposit and dissemination of scientific research documents, whether they are published or not. The documents may come from teaching and research institutions in France or abroad, or from public or private research centers.
L'archive ouverte pluridisciplinaire HAL, est destinée au dépôt et à la diffusion de documents scientifiques de niveau recherche, publiés ou non, émanant des établissements d'enseignement et de recherche français ou étrangers, des laboratoires publics ou privés. 


\title{
Exploitation du cheval au Magdalénien supérieur dans le Sud-Ouest de la France
}

\author{
Le cas de l'abri Faustin (Cessac, Gironde)
}

\author{
Léa Feyfant, David Cochard et Jean-Baptiste Mallye
}

Résumé : Le Magdalénien supérieur se développe durant la fin de l'épisode d'Heinrich 1 (Dryas ancien) et l'interstade du Bölling. Cette période est marquée par une recomposition des biotopes et la réouverture de territoires pour les groupes humains. Cette culture connaît dorénavant une extension à l'échelle de l'Europe et plusieurs modèles de mobilités territoriales sont proposés dans différentes régions (Bassin parisien, plateau Suisse, Rhénanie). Ces modèles sont basés sur l'alternance saisonnière de chasses spécialisées sur le renne et le cheval ou de chasses de ces ongulés en proportion égale. Dans le Sud de la France, les spectres de chasse se diversifient au profit d'ongulés forestiers ou montagnards mais également de petits gibiers. Les chasses spécialisées y sont exceptionnelles. L'abri Faustin, situé dans le Nord du Bassin aquitain, est un des rares gisements du Sud-Ouest de la France appartenant au Magdalénien supérieur ayant livré un spectre faunique majoritairement dominé par le cheval. Malgré cette singularité, ce gisement n'a jamais fait l'objet d'une étude archéozoologique détaillée. De nouvelles datations récemment effectuées dans le cadre du projet « Magdatis » ont permis de confirmer une occupation contemporaine de l'interstade du Bölling. Nous proposons ici une étude détaillée des stratégies de prédation, de transport ainsi que des pratiques bouchères employées sur le cheval au cours du Magdalénien supérieur. L'étude taphonomique a mis en évidence une excellente préservation des restes osseux et dentaires permettant la mise en œuvre d'une étude archéozoologique à haute résolution. Par ailleurs, les remontages montrent une dispersion limitée des vestiges. Le cheval est l'espèce dominante en termes de nombre de restes et de quantité de ressources, même si son exploitation ne marque pas une spécialisation dans les activités pratiquées sur le site. Les Magdaléniens ont privilégié les groupes familiaux, plus prévisibles, aux groupes de célibataires. Les indices de saisonnalité révèlent que les épisodes de chasse se sont répartis tout au long de l'année. La présence de toutes les portions squelettiques sur le gisement et de restes de fœtus va dans le sens d'un transport de carcasses entières. Cela nous amène à conclure que le site d'abattage devait se trouver dans les environs immédiats de l'abri. La reconstruction de la chaîne opératoire de boucherie montre que le cheval a été exploité pour ses ressources alimentaires (viandes, moelles et graisses) mais également utilitaires. Les résultats de notre étude révèlent de nombreux points de convergence avec les gisements dominés par le cheval et contemporains de l'occupation de l'abri Faustin en Rhénanie, sur le plateau Suisse et dans le Bassin parisien (Bignon, 2003; Street et al., 2006; Müller, 2014) : saison et tactique de chasse, proximité du lieu d'abattage et du lieu de traitement des carcasses, exploitation à des fins alimentaires et utilitaires. Ces observations vont dans le sens d'une certaine homogénéité dans le mode d'exploitation de cet ongulé à l'échelle de l'Europe pour le Magdalénien supérieur.

Mots clés : Magdalénien, Tardiglaciaire, stratégies de subsistance, Equus caballus, Bassin aquitain.

Abstract: The Late Magdalenian coincides with the end of the Heinrich 1 (Oldest Dryas) event and the Bölling interstadial. This period was marked by the recomposition of biotopes and the reopening of territories to human groups. The culture began to expand throughout Europe and territorial mobility models have been proposed for several regions (Paris Basin, Swiss Plateau, Rhineland). These models are based on the seasonal alternation of specialized hunting of reindeer and horse or the hunting of these ungulates in equal proportions. In the south of France, hunting was more diversified and included greater proportions of forest and mountain ungulates and small game. Specialized hunting was rare. Abri Faustin, situated in north of the Aquitaine Basin, is one of the few sites in the south west of France dating to the Late Magdalenian with a faunal spectrum dominated principally by horse. Despite this particularity, the site has never been subject to a detailed archaeozoological study.

New datings recently performed as part of the Magdatis project have confirmed an occupation contemporary with the Bölling interstadial. We here propose a detailed study of the hunting strategies, transport, and butchery practices carried out on horses at this site. The methods that were used are conventional in zooarchaeology: age determination, quantification of skeletal elements and observation and quantification of natural and anthropogenic marks. The bone assemblage contains 1453 bone and dental remains. A taphonomic study has revealed the excellent preservation of these remains, allowing a high-resolution archaeozoological study to be performed. Moreover, the bone refits shows that the dispersal of the remains is limited. Horse is the dominant species in terms of the number of remains (NISP 
$=853)$, number of specimens $(\mathrm{MNI}=20)$ and quantity of resources. Its exploitation does not appear to represent a specialization in terms of the activities carried out on the site in view of the food and utilitarian exploitation of small game (Le Bail, 2005; Mallye, 2007; Val, 2009) and the presence of domestic lithic and bone tools. The Magdalenians favoured family groups, which were more predictable, rather than singles groups. Seasonality indices show that hunting episodes took place throughout the year. Hunting of singles groups did occur but was more opportunistic than that of family groups. Hunting strategy can be presumed but not clearly demonstrated. We presume that the Magdalenian took advantage of the topography of Entre-deux-Mers to approach the groups of horses.

High disparities in skeletal representation cannot be explained by differential preservation and food utility indices (FUI and MUI) are not correlated with the survival percentage. We assume that whole horse carcasses were transported from the kill site to the base camp. The presence of foetal remains supports this hypothesis. This leads us to conclude that the kill site must have been located in the immediate vicinity of the shelter. The reconstruction of the butchery processes implies that horses were exploited both for their food resources (meat, marrow, and fat), and for utilitarian and symbolic purposes. We propose the use of a bone boiling method for the extraction of marrow from spongy shafts and of fat from long bone ends. The results of our study reveal many common elements with sites dominated by Horse, contemporary with the occupation of Abri Faustin, in the Rhineland, on the Swiss Plateau, and in the Paris Basin (Bignon, 2003; Street et al. 2006; Müller, 2014): seasons and hunting strategies, proximity of the kill and processing sites, exploitation for food and utilitarian and symbolic purposes. Carcass exploitation was always optimal, with extraction of fat and marrow from mandible and long bones. Even if the use of horse bones for utilitarian and symbolic purposes was never systematic in the Late Magdalenian, we see it sporadically on some sites. All these observations suggest a certain uniformity in the exploitation of this ungulate across Europe during the Late Magdalenian.

Keywords: Magdalenian, Late Glacial, Subsistence strategies, Equus cabalus, Aquitaine Basin.

L

E Magdalénien supérieur daté entre environ 16500 et 14000 cal. BP, coïncide avec la fin de l'épisode d'Heinrich 1 (sensu Stanford et al., 2011) et l'interstade du Bölling (i. e. GI-1e, Rasmussen et al., 2014). Cette période est caractérisée par un réchauffement climatique entrainant une recomposition des biocénoses (e.g. Delpech, 1999; Bridault et Chaix, 2009; Langlais et al., 2012) et la réouverture de territoires (e. g. Demars, 2002). Parallèlement à ces changements environnementaux, les comportements de subsistance des chasseurs-cueilleurs magdaléniens évoluent. Cela va se refléter tant dans leur équipement que dans leur tableau de chasse (Langlais et al., 2012 et 2014). Dans le Sud de la France, si le renne, le cheval et les grands Bovinés se retrouvent en abondance dans certains gisements comme à Duruthy, Landes, Dufaure, Landes, Bize, Aude, le Morin, Gironde, gare de Couze, Dordogne (e. g. Delpech, 1967 et 1975; Fontana, 1998 et 1999; Moigne, 2003; Costamagno, 2006), la proportion d'autres espèces comme le cerf ou le bouquetin va augmenter significativement jusqu'à devenir prépondérante, dans les spectres fauniques, en particulier dans les Pyrénées, comme à Arancou, Pyrénées-Atlantiques, Troubat, Hautes-Pyrénées, les Églises, Ariège, la Vache, Ariège, Belvis, Aude ou encore les Conques, PyrénéesOrientales (Delpech et Villa, 1993; Pailhaugue, 1995 et 1998; Fontana, 1998 et 1999; Costamagno, 2005 et 2006). À cela s'ajoute l'exploitation des petits gibiers tels que les oiseaux, les Léporidés, les petits carnivores ou encore les poissons (Fontana, 1998; Le Gall 2003; Cochard, 2004; Cochard et Brugal, 2004; Costamagno et Laroulandie, 2004; Costamagno et al., 2008; Laroulandie, 2009; Langlais et al., 2012 et 2014; Laroulandie et al., 2014).

Dans le Nord de l'Europe, plusieurs modèles de mobilités territoriales sont proposés sur la base du rôle prédominant et conjoint du cheval et du renne (Weniger, 1987; Bignon, 2003; Müller, 2006; Street et al., 2012). En particulier, des hypothèses de mobilité saisonnière s'appuient sur l'existence de gisements témoignant de grandes chasses collectives automnales sur le renne comme à Pincevent niveau IV20, Seine-et-Marne, Verberie, Oise, au Petersfels, Baden-Württemberg, Allemagne (Berke, 1992; Enloe et David, 1997; Enloe, 2000) et de la chasse du cheval toute l'année, avec un pic au printemps : Andernach, Gönnersdorf, Rhénanie, Allemagne, Hauterive-Champréveyres, Monruz, Neuchâtel, Suisse, Tureau-des-Gardes, Grand Canton, Seine-et-Marne (Street, 1997; Morel et Müller, 1997; Bignon, 2003; Street et Turner, 2013; Müller, 2014). Dans la moitié sud de la France, même si le renne reste dominant sur de nombreux gisements - La Madeleine, Dordogne, Grande Bize, Aude, Magdeleine La Plaine, Tarn (Boyle, 1994; Fontana, 1998; Kuntz, 2006) -, il ne semble pas faire l'objet de grandes chasses collectives automnales (Costamagno, 2003; Kuntz et Costamagno, 2011). Quant au cheval, il n'existe de chasses " spécialisées » qu'à Solutré, Saône-et-Loire où cette pratique semble avoir été transmise sur plusieurs générations (Turner, 2002). En Gironde, deux gisements montrent un cortège dominé par les restes osseux de chevaux et de rennes : le Morin (Kuntz in Mallye et al., en préparation) et l'abri Faustin (Delpech, 1967, 1971 et 1975). Dans ce dernier, le cheval domine le spectre faunique et cette singularité en fait un cas idéal pour comprendre, d'une part, les modalités d'exploitation de ce grand herbivore et, d'autre part, son statut socio-économique dans le Nord du Bassin aquitain.

Il s'agit alors de définir les stratégies de prédation mises en œuvre, les modalités de transport ainsi que les pratiques bouchères réalisées par les Magdaléniens. Il s'avère toutefois nécessaire de vérifier au préalable l'homogénéité et l'intégrité du gisement, et notamment 
l'impact des processus de formation du site sur l'assemblage osseux. Une étude taphonomique a été réalisée à cet effet.

\section{PRÉSENTATION DU GISEMENT}

C e gisement de l'abri Faustin est situé dans la vallée de Ll'Engranne, dans l'Entre-deux-Mers, sur la commune de Cessac, Gironde, au lieu dit « Barreaux » (fig. 1). L'abri effondré est creusé dans une petite falaise de calcaire à astéries exposée au sud. S. Terraza et M. Grousset effectuèrent un sondage dans les années 1950. Puis, M. Lenoir et S. Terraza entreprirent trois campagnes de fouilles, de 1975 à 1977, au cours desquelles une surface de $25 \mathrm{~m}^{2}$ fut fouillée. Ces campagnes firent l'objet de techniques de fouilles modernes : carroyage, tamisage à l'eau, relevés des coordonnées et études stratigraphiques.

Le remplissage, découpé en trois lithofaciès décrits par M. Lenoir (1983), résulterait de l'effondrement du porche et du plancher de l'abri. Le matériel archéologique, réparti sur l'ensemble de la stratigraphie, est apparu homogène sur la totalité du dépôt (Lenoir, 1983).

L'industrie lithique est caractérisée par la présence d'éléments du Magdalénien supérieur tels que des pointes à cran, des pointes de Laugerie-Basse et des triangles scalènes; ainsi que de rares éléments aziliens, avec notamment des pointes aziliennes (Lenoir et Terraza, 1971; Lenoir, 1983). L'industrie osseuse est composée de plusieurs harpons magdaléniens à deux rangs de barbelures, auxquels s'associent des sagaies, ciseaux, baguettes, poinçons et aiguilles en os ainsi que des déchets de fabrication (Lenoir, 1983). M. Lenoir voyant dans ce matériel le processus

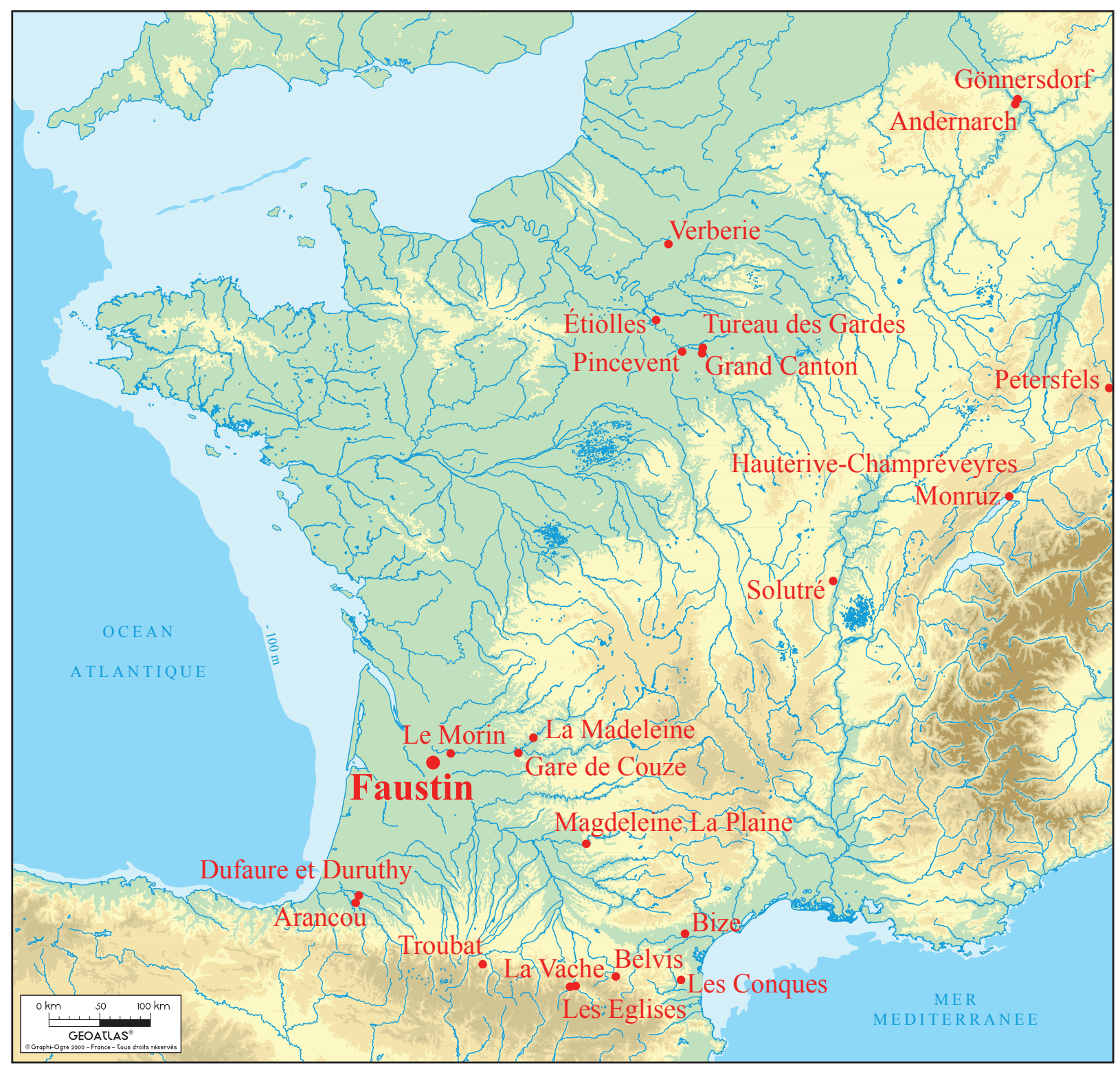

Fig. 1 - Abri Faustin. Localisation de l'abri et des sites magdaléniens cités dans le texte.

Fig. 1 - Abri Faustin. Location of the shelter and the Magdalenian sites mentioned in the text. 
d'azilianisation auparavant décrit à l'abri Morin et à Gare de Couze (Bordes et Sonneville-Bordes, 1979) attribua ainsi l'occupation au Magdalénien final (Lenoir, 1983).

La faune issue du sondage Terraza et Grousset a été décrite par Delpech (1971 et 1975). Au total 153 restes ont été déterminés, le cheval (Equus caballus) représentant $30 \%$ du nombre de restes déterminés (NRD) toutes espèces confondues et presque $50 \%$ du NRD des grands herbivores. L'espèce la mieux représentée après le cheval est la chouette harfang (Bubo scandiacus ; $25 \%$ du NRD). Ce matériel n'est malheureusement plus disponible dans son intégralité car une partie a été égarée au cours d'un déménagement.

Plusieurs études archéozoologiques ont été menées sur les collections Lenoir et Terraza. Celles-ci concernent exclusivement la petite faune. Les restes de blaireau (Meles meles), par ailleurs absents de la collection ancienne (voir Delpech, 1971 et 1975), ont été étudiés par l'un d'entre nous (Mallye, 2007); les restes de harfang et de renard (Vulpes vulpes) ont fait l'objet d'études archéozoologiques respectivement par A. Le Bail (2005) et A. Val (2009). Ces trois études mettent en évidence l'acquisition et la consommation par l'homme de ces petits gibiers.

Cinq datations radiocarbone sont actuellement disponibles pour discuter de l'occupation humaine de l'abri : une date conventionnelle effectuée sur un lot de restes osseux et quatre datations en AMS réalisées dans le cadre du projet « Magdatis » à partir d'ossements déterminés spécifiquement (tabl. 1; fig. 2).

Toutes ces dates sont cohérentes entre elles. Cependant, la calibration révèle qu'elles se situent sur une zone de plateau radiocarbone. Ce dernier entraîne un étalement des dates une fois calibrées. Malgré cela, l'occupation de l'abri Faustin par les Magdaléniens est relativement bien délimitée puisque centrée sur le Bölling.

\section{MATÉRIELS}

T e matériel étudié est issu de la travée 24 et des bandes $\mathrm{K}$, L et $\mathrm{M}$ des collections Lenoir et Terraza (fig. 3). Ces $3 \mathrm{~m}^{2}$ représentent environ un huitième de la surface fouillée. Cette travée a été choisie en raison de sa richesse en matériel. Les restes d'ongulés déterminés spécifiquement ont été isolés et décomptés afin d'apprécier la diversité du spectre des grands ongulés. La mésofaune n'a pas été considérée du fait de l'échantillonnage induit par ses études antérieures. L'étude archéozoologique menée ici porte uniquement sur les restes d'Équidés. Les restes non déterminés ainsi que ceux appartenant aux autres ongulés ont néanmoins été décomptés et pris en compte dans l'analyse taphonomique. Lorsque cela était possible, une classe de taille d'herbivores (voir Costamagno, 1999) a été attribuée aux restes qui ne pouvaient pas être déterminés spécifiquement. Enfin, les restes dentaires de chevaux issus de la travée 25 ont été intégrés à l'étude afin d'optimiser les opérations de remontage des séries dentaires et le calcul du nombre minimal d'individus (NMI). La travée 25 a été choisie en raison de sa position (adjacente à la travée 24) et de sa richesse en matériel dentaire.

\begin{tabular}{|l|c|c|c|}
\multicolumn{1}{l|}{ Codes laboratoire } & Dates non calibrées (BP) & Dates calibrées (cal. BP) \\
\hline Lot d'os & Ly 2700 & $12370 \pm 220$ & $15621-13566$ \\
\hline Bos / Bison & OxA 26 660 & $12415 \pm 50$ & $15029-14117$ \\
\hline Cervus & OxA 26 661 & $12325 \pm 50$ & $14840-14019$ \\
\hline Equus & Ly 10 177 (SacA 32 844) & $12370 \pm 60$ & $14996-14060$ \\
\hline Rangifer & Poz-52974 & $12420 \pm 70$ & $15088-14095$ \\
\hline
\end{tabular}

Tabl. 1 - Abri Faustin. Présentation du corpus de dates radiocarbone.

Table 1 - Abri Faustin. Presentation of the corpus of radio carbon dates.

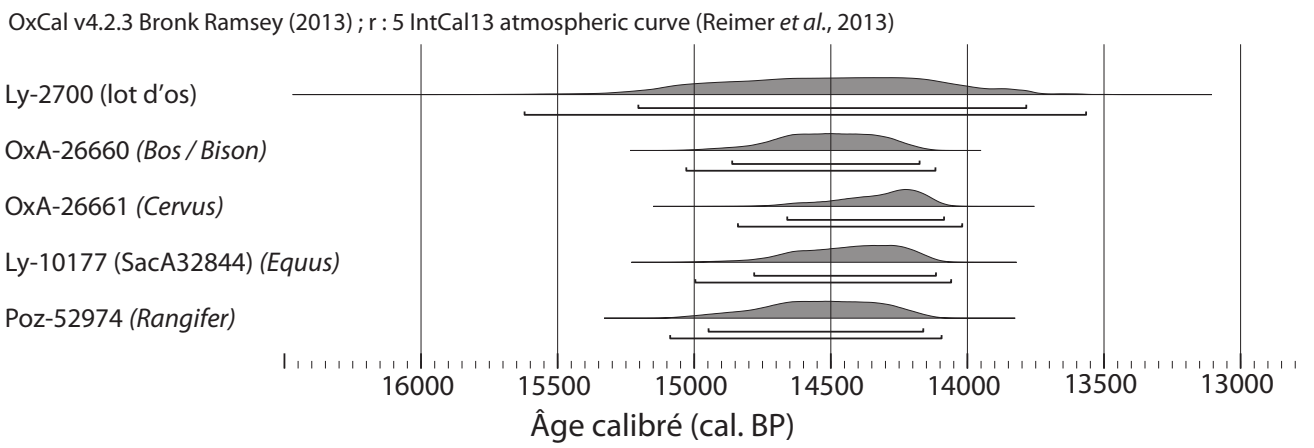

Fig. 2 - Abri Faustin. Courbes de calibration des dates ${ }^{14} \mathrm{C}$.

Fig. 2 -Abri Faustin. Calibration curve for ${ }^{14} \mathrm{C}$ dating. 


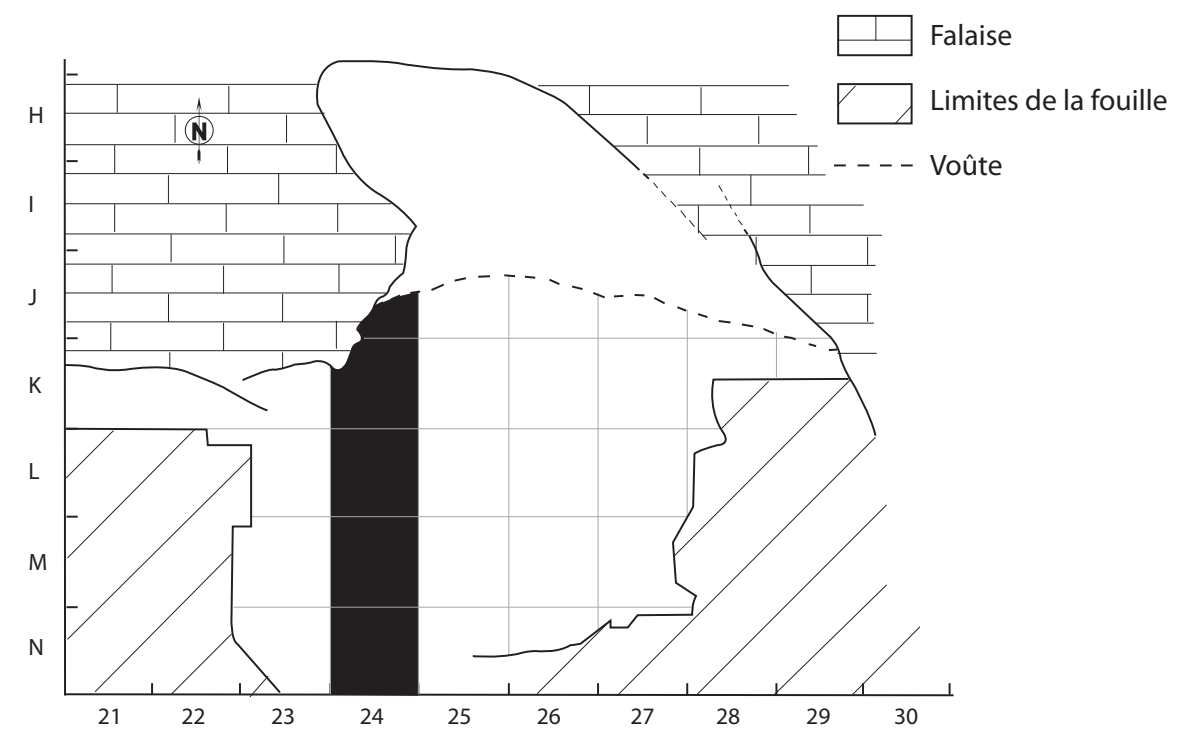

Fig. 3 - Abri Faustin. Vue sagittale de l'abri d'après Lenoir, 1983, modifié. La zone en noir correspond à la travée étudiée.

Fig. 3 - Abri Faustin. Sagittal view of the shelter by Lenoir, 1983, modified. The zone in black corresponds to the sector studied.

\section{MÉTHODES}

\section{Quantification et remontages}

Le nombre de restes déterminés (NRD) est utilisé pour quantifier l'abondance taxonomique de l'assemblage osseux. Le dénombrement de la population équine est basé sur le nombre minimal d'individus (NMI). Ce dernier est établi sur le NMI de combinaison (NMIc), après remontages en prenant en compte les critères de taille, de maturation et d'usure dentaire. Le remontage des séries dentaires est effectué selon le protocole mis en ouvre par Morel et Müller (1997) puis par Bignon et Müller (in Bignon, 2003). Dans un premier temps, des remontages entre les fragments dentaires sont recherchés. Des raccords sont ensuite menés entre les dents dont les surfaces de contacts assurent une connexion idéale sur l'ensemble du bord. Lorsque les dents sont trop abîmées pour permettre une reconnexion parfaite, des rapprochements d'ordre 1 sont effectués en se basant sur la hauteur et la forme des couronnes, le degré d'usure, le dessin de la surface occlusale et les anomalies dentaires d'origine pathologique ou non. Enfin, certaines dents, isolées et très mal conservées, font l'objet de rapprochements d'ordre 2, uniquement sur la base de la hauteur de la couronne et de l'usure dentaire. Ces remontages ont été effectués indépendamment par deux d'entre nous (L. F. et J.-B. M.) afin d'avoir un regard croisé permettant de minimiser la subjectivité de la démarche. Cette approche a permis d'améliorer l'estimation du NMI et de préciser l'âge au décès des individus.

\section{Fréquence des parties squelettiques}

La connaissance des fréquences des parties squelettiques permet d'aborder des questions tant d'ordre tapho- nomique qu'archéozoologique notamment concernant les modalités de transport des carcasses. Pour documenter la représentation osseuse, nous choisissons d'utiliser le nombre minimal d'éléments, NME (Lyman, 1994a) et le pourcentage de survie (Brain, 1969).

Afin d'évaluer l'impact de la conservation différentielle sur l'ensemble osseux, le pourcentage de survie de chaque portion des éléments squelettiques est comparé avec la valeur de la densité osseuse pour chacune de ces zones (Lyman, 1994b) grâce à un test de Spearman rho. Les valeurs de densité utilisées pour le cheval proviennent du travail de Lyman (1994a) et révisées par Lam et al. (1998 et 2003).

Les questions relatives aux stratégies de transport des carcasses et plus généralement de la fonction du site sont abordées en comparant les fréquences des parties du squelette avec leur indice d'utilité nutritive (Binford, 1978). Les valeurs d'utilités (FUI : Food Utility Index; MUI : Marrow Utility Indice) sont issues de Outram et Rowley-Conwy (1998). Là encore, le test de Spearman rho nous permet d'évaluer la relation statistique entre ces valeurs et la fréquence des parties squelettiques (pourcentage de survie).

\section{Lecture des surfaces osseuses}

Les surfaces osseuses peuvent être affectées par des modifications naturelles ou anthropiques. Afin de mesurer l'impact de ces agents taphonomiques (sensu Lyman, 1994b; Fisher, 1995) sur l'ensemble osseux, chaque reste est examiné à l'aide d'une loupe binoculaire de grossissement variable $(\times 10$ à $\times 40)$. L'étendue des surfaces altérées est quantifiée et codée de la façon suivante dans la base de données :

$0=0 \%$ de la surface lisible de la pièce;

$1=$ moins de $25 \%$;

$2=$ entre 25 et $50 \%$ exclus;

$3=$ entre 50 et $75 \%$;

$4=75 \%$ et plus. 
Cela permet de relativiser la fréquence des traces anthropogéniques en fonction de l'état des surfaces osseuses. Celles-ci (stries de découpe, de raclage, impacts de percussion, etc.) sont systématiquement reportées sur des planches anatomiques afin d'étudier et d'interpréter leur distribution spatiale.

La fréquence des stries de découpe (NRDcut/NRD : rapport entre le nombre de restes déterminés portant des traces de découpe et le nombre de restes déterminés) est généralement utilisée pour appréhender l'impact anthropique sur l'ensemble osseux (Bunn, 1982). La reconnaissance des différentes étapes d'exploitation des animaux et des produits recherchés (alimentaire et technique) par les chasseurs-cueilleurs ainsi que les moyens mis en œuvre pour les obtenir est réalisée grâce à l'analyse des traces anthropogéniques. Leur localisation, leur orientation et la récurrence de leur position permettent d'identifier les différentes étapes de la chaîne opératoire de boucherie au regard de référentiels expérimentaux. Pour le cheval, à notre connaissance, aucune boucherie expérimentale n'a pour l'instant été réalisée. L'interprétation des marques relevées sur les chevaux de Faustin s'est donc basée sur les référentiels établis sur le renne (Binford, 1981; Nilssen, 2000; Costamagno et David, 2009). Nous intégrons également à notre réflexion les analyses détaillées de l'exploitation des carcasses de chevaux des sites magdaléniens de Monruz et Hauterive-Champréveyres (Morel et Müller, 1997; Müller, 2014). Les traces de chauffe et de combustion observées sur les ossements nous permettent, quant à elles, de discuter des procédés de préparation des produits alimentaires.

\section{Fragmentation et fracturation}

La morphologie des bords de fracture des os longs est analysée en utilisant une typologie dérivée de la méthode de Villa et Mahieu (1991). Nous différencions ainsi les cassures sur os secs, résultant de la fragmentation naturelle, des cassures sur os frais, résultant de la fracturation (boucherie ou charognage). Ces observations, combinées à l'identification de traces d'origine anthropique (impacts de percussion) ou naturelles (carnivores), permettent non seulement d'identifier l'agent responsable de la fracturation, mais également de mettre en évidence une étape de la chaîne opératoire de boucherie, en l'occurrence la récupération de la moelle. Enfin, la double patine, témoignant d'une cassure récente liée à la fouille ou consécutive à une fragmentation lors du stockage du matériel, est également prise en compte.

\section{Populations chassées}

Afin de comprendre les stratégies de chasse mises en œuvre, il est essentiel de connaître le comportement écoéthologique des espèces-proies. En ce qui concerne les chevaux sauvages deux groupes sociaux se distinguent (Groves, 1974) : les harems, ou groupes familiaux, composés d'un étalon, de cinq à six juments et de leurs poulains jusqu'à deux ans et le groupe de célibataires, composé uniquement de mâles adultes et d'anciens étalons.

La détermination de l'âge au décès des chevaux adultes est appréhendée par la méthode développée par Fernandez et Legendre (2003) reposant sur la dynamique d'usure des

\begin{tabular}{|c|c|c|c|c|c|}
\hline Détermination de l'âge & Classe $0-2$ ans & Classe 2-5 ans & Classe $5-10$ ans & Classe $10-15$ ans & Classe +15 ans \\
\hline $\begin{array}{l}\text { Usure des dents déciduales + } \\
\text { éruption des M } 1 / 2\end{array}$ & $\begin{array}{c}\text { Juvéniles } \\
\text { liés aux groupes } \\
\text { familiaux }\end{array}$ & & & & \\
\hline $\begin{array}{l}\text { Remplacement des dents } \\
\text { déciduales }+ \text { éruption des } \\
\text { PM2/3/4 et M3 }\end{array}$ & & $\begin{array}{c}\text { Subadultes } \\
\text { exil des mâles } \\
\text { hors des GF vers } \\
\text { les GC }\end{array}$ & & & \\
\hline $\begin{array}{l}\text { Usure des dents jugales } \\
\text { définitives }\end{array}$ & & & $\begin{array}{c}\text { Adultes dans } \\
\text { la pleine force } \\
\text { de l'âge } \mathrm{GF}= \\
\text { juments + étalon } \\
\text { GC = étalons } \\
\text { divers }\end{array}$ & & \\
\hline $\begin{array}{l}\text { Usure des dents jugales } \\
\text { définitives }\end{array}$ & & & & $\begin{array}{c}\text { Vieux adultes } \\
\text { GF = juments } \\
\text { GC = étalons, } \\
\text { parfois solitaires }\end{array}$ & \\
\hline $\begin{array}{l}\text { Usure des dents jugales } \\
\text { définitives }\end{array}$ & & & & & $\begin{array}{c}\text { Très vieux adultes } \\
\text { GF }=\text { juments } \\
\text { GC }=\text { étalons, } \\
\text { parfois solitaires }\end{array}$ \\
\hline
\end{tabular}

Tabl. 2 - Classes d'âge établies pour la courbe de mortalité des chevaux (d'après Bignon, 2003, modifié). GF = groupes familiaux; $\mathrm{GC}=$ groupes de célibataires.

Table 2 - Age groups established for the mortality curve of horses (after Bignon, 2003, modified). GF = family group; GC = singles groups. 
dents permanentes. Pour la détermination de l'âge des poulains, nous utilisons la méthode développée par O. Bignon (2003 et 2006) à partir des dents jugales déciduales. Cette méthode repose sur le même principe que celle de Fernandez et Legendre (2003) et fournit un âge avec un intervalle de confiance d'un mois correspondant au pic de naissance des chevaux (mai). L'âge des fœtus est déterminé grâce aux tables fournies par Prummel (1987, 1988 et 1989).

Enfin, la répartition des individus par classes d'âges suit celle décrite par O. Bignon (2003; ici tabl. 2).

\section{RÉSULTATS}

\section{Spectre des ongulés}

L'échantillon issu de la travée 24 comporte 1453 restes osseux et dentaires d'ongulés dont 879 sont déterminés anatomiquement et spécifiquement. Le cheval (tabl. 3) est de loin le plus abondant avec $97 \%$ des restes déterminés $(\mathrm{NRD}=853$ restes). Les autres grands herbivores représentés sont, dans l'ordre décroissant, le renne $(\mathrm{NRD}=10)$, les Bovidés $(\mathrm{NRD}=8)$, le cerf $(\mathrm{NRD}=5)$, le chamois $(\mathrm{NRD}=2)$ et le mammouth représenté par un fragment de mandibule. Parmi les restes non déterminés spécifiquement, les ongulés 3/4 (Équidés, grands Bovidés, cerf...; NR = 429) sont les mieux représentés, suivis dans l'ordre décroissant des classes $2 / 3(\mathrm{NR}=32)$ et $2(\mathrm{NR}=5)$. Un peu plus d'une centaine de restes n'ont pas été attribués à une classe de taille d'ongulés.

\section{Altération des surfaces osseuses}

La quantification du degré de lisibilité des surfaces osseuses révèle que l'altération liée aux phénomènes

\begin{tabular}{|c|c|c|}
\hline Taxons & NR & $\%$ NRD \\
\hline Equus caballus & 853 & $97,0 \%$ \\
\hline Rangifer tarandus & 10 & $1,1 \%$ \\
\hline Bos/Bison & 8 & $0,9 \%$ \\
\hline Cervus elaphus & 5 & $0,6 \%$ \\
\hline Rupicapra rupicapra & 2 & $0,2 \%$ \\
\hline Mammuthus primigenius & 1 & $0,1 \%$ \\
\hline Ongulé 3/4 & 429 & \\
\hline Ongulé 2/3 & 32 & \\
\hline Ongulé 2 & 5 & \\
\hline Ongulé indéterminé & 108 & \\
\hline Total & 1453 & \\
\hline Total NRD & 879 & \\
\hline
\end{tabular}

Tabl. 3 -Abri Faustin. Nombre de restes par taxons et par classe de taille d'ongulé pour la travée 24 .

Table 3 - Abri Faustin. Number of specimens per taxon and per ungulate size class for sector 24. taphonomiques est relativement importante. En effet, un peu plus du quart du matériel $(25,8 \%)$ présente une surface totalement illisible. Malgré tout, $43 \%$ du matériel est bien lisible (plus de $50 \%$ de la surface non altérée) et $21 \%$ assez bien lisible (entre 25 et $50 \%$ de la surface lisible). Les principaux agents responsables de ces altérations naturelles sont les racines, le weathering et la dissolution (tabl. 4). Aucune trace de carnivore n'est identifiée et les traces de rongeurs sont extrêmement rares $(0,4 \%)$. Le weathering affecte 38,5\% de l'assemblage. Dans $95 \%$ des cas, il ne s'agit que de légères fissures peu profondes (stade 1). Le décollement de fines lamelles d'os - rencontré dans seulement $3 \%$ des cas (stade 2) - et les attaques plus sévères sont, anecdotiques. La dissolution affecte $22,2 \%$ sous la forme de petites cupules à la surface des os. Les traces de racines sont observées sur un peu plus de la moitié du matériel. Elles sont le principal phénomène responsable d'une perte d'information potentielle. Enfin, le recouvrement des restes par de la calcite reste très négligeable.

\section{Fragmentation vs fracturation}

Le pourcentage d'os complets dans l'assemblage est relativement faible puisque seulement $14 \%$ des vestiges sont entiers, principalement les os du carpe et les sésamoïdes (tabl. 5). L'étude de la morphologie des bords de fracture des os longs permet de distinguer trois types de cassures imputables à autant de mécanismes de cassure des os. Les cassures affectant les diaphyses sont produites majoritairement sur os frais (plus 75\% des cas). 6,5\% des vestiges portent des impacts de percussion. La fracturation sur os frais semble donc être le résultat exclusif d'actions humaines. Les cassures sur os secs, dues à des phénomènes post-dépositionnels, concernent près de $21 \%$ des cassures observées. Notons qu'une petite partie des vestiges $(4 \%)$ atteste d'une fragmentation récente. Les extré-

\begin{tabular}{|l|c|}
\hline Phénomènes observés & $\% \mathrm{du}$ NR \\
\hline Racines & $52,9 \%$ \\
\hline Weathering & $38,5 \%$ \\
\hline Stade 1 & $96,7 \%$ \\
\cline { 2 - 2 } Stade 2 & $3,1 \%$ \\
\cline { 2 - 2 } Stade 3 & $0,2 \%$ \\
\hline Dissolution & $22,2 \%$ \\
\hline Concrétionnement & $0,5 \%$ \\
\hline Traces de rongeurs & $0,4 \%$ \\
\hline Hommes & $23,7 \%$ \\
\hline Cut marks & $21,5 \%$ \\
\cline { 2 - 2 } Percussion & $4,5 \%$ \\
\hline
\end{tabular}

Tabl. 4 - Abri Faustin. Processus taphonomiques observés en pourcentage du nombre de restes total (NR) de la travée 24 .

Table 4 - Abri Faustin. Taphonomic processes observed as a percentage of the total number of specimens (NSP) in sector 24. 


\begin{tabular}{|c|c|c|c|c|c|c|}
\hline \multicolumn{2}{|l|}{ Éléments } & \multirow{2}{*}{$\begin{array}{c}\text { NRD } \\
274\end{array}$} & \multirow{2}{*}{$\begin{array}{c}\text { NME } \\
-\end{array}$} & \multirow{2}{*}{$\begin{array}{c}\% \text { survie } \\
-\end{array}$} & \multirow{2}{*}{$\begin{array}{c}\% \text { os complets } \\
31,8\end{array}$} & \multirow{2}{*}{$\begin{array}{c}\text { NRDcut } \\
-\end{array}$} \\
\hline Dents & $\mathrm{TH}$ & & & & & \\
\hline Canines & & 3 & - & - & - & - \\
\hline Incisives & & 48 & - & - & - & - \\
\hline Jugales supéieures & & 68 & - & - & - & - \\
\hline Jugales inférieures & & 75 & - & - & - & - \\
\hline Jugales & & 80 & - & - & - & - \\
\hline Crâne & SK & 80 & 3 & $25,0 \%$ & 0,0 & 12 \\
\hline Rocher & Roc. & 0 & - & - & - & - \\
\hline Mandibule & Mand & 122 & 8 & $66,7 \%$ & 0,0 & 36 \\
\hline Hyoïde & Hyo. & 2 & 1 & $16,7 \%$ & 0,0 & 1 \\
\hline Atlas & AT & 5 & 1 & $16,7 \%$ & 0,0 & 4 \\
\hline Axis & $\mathrm{AX}$ & 0 & - & - & 0,0 & - \\
\hline Vetrèbres cervicales (3 à 7) & $\mathrm{CE}$ & 8 & 2 & $6,7 \%$ & 0,0 & 2 \\
\hline Vertèbres thoraciques & $\mathrm{TH}$ & 8 & 3 & $2,8 \%$ & 0,0 & 3 \\
\hline Vertèbres lombaires & LU & 1 & 1 & $2,4 \%$ & 0,0 & 1 \\
\hline Vertèbres NID & - & 4 & - & - & - & 0 \\
\hline Sacrum & $\mathrm{SC}$ & 0 & - & - & - & - \\
\hline Vertèbres caudales & Caud. & 0 & - & - & - & - \\
\hline Côtes & RI & 69 & 1 & $0,5 \%$ & 0,0 & 32 \\
\hline Scapula & SP & 0 & - & - & - & - \\
\hline Humérus & $\mathrm{HU}$ & 20 & 5 & $41,7 \%$ & 0,0 & 14 \\
\hline Proximal & HU1 & 5 & 1 & $8,3 \%$ & - & \\
\hline Diaphyse & HU2 & 6 & 5 & $41,7 \%$ & - & \\
\hline Distal & HU3 & 10 & 3 & $25,0 \%$ & 20,0 & \\
\hline Radius-ulna & RA & 27 & 5 & $41,7 \%$ & 7,4 & 12 \\
\hline Proximal & RA1 & 5 & 4 & $33,3 \%$ & 40,0 & \\
\hline Diaphyse & RA2 & 20 & 4 & $33,3 \%$ & 10,0 & \\
\hline Distal & RA3 & 5 & 4 & $33,3 \%$ & 0,0 & \\
\hline Triquetrum & Triq. & 1 & 1 & $8,3 \%$ & 100,0 & 1 \\
\hline Lunatum & Lun. & 3 & 3 & $25,0 \%$ & 33,3 & 0 \\
\hline Scaphoïde & Scaph & 1 & 1 & $8,3 \%$ & 100,0 & 0 \\
\hline Hamatum & Ham. & 0 & - & - & - & - \\
\hline Capitatum & Capi. & 2 & 2 & $16,7 \%$ & 100,0 & 1 \\
\hline Trapezoïde & Trap. & 2 & 2 & $16,7 \%$ & 100,0 & 0 \\
\hline Pisiforme & Pisi. & 1 & 1 & $16,7 \%$ & 100,0 & 0 \\
\hline Métacarpiens 3 & $\mathrm{MC}$ & 16 & 4 & $33,3 \%$ & 0,0 & 5 \\
\hline Proximal & $\mathrm{MC} 1$ & 3 & - & $25,0 \%$ & 0,0 & \\
\hline Diaphyse & $\mathrm{MC} 2$ & 12 & - & $33,3 \%$ & 0,0 & \\
\hline Distal & MC3 & 4 & - & $33,3 \%$ & 75,0 & \\
\hline Métacarpiens accessoires & Mcacc & 0 & - & - & - & - \\
\hline Ischium & IS & 2 & 2 & $33,3 \%$ & 0,0 & 1 \\
\hline Ilium & IL & 0 & - & - & - & - \\
\hline Acétabulum & $\mathrm{AC}$ & 0 & - & - & - & - \\
\hline
\end{tabular}

Tabl. 5 - Abri Faustin. Nombre de restes déterminés (NRD), nombre minimal d'éléments (NME), pourcentages de survie et nombre de restes déterminés portant des stries de découpe (NRDcut) pour chaque élément squelettique de cheval de la travée 24.

Table 5 - Abri Faustin. Number of identified specimens (NISP), minimum number of elements (MNE), survival-percentage rates, and number of identified specimens displaying cut marks (NISPcut) for each horse skeletal element in sector 24. 


\begin{tabular}{|c|c|c|c|c|c|c|}
\hline \multicolumn{2}{|l|}{ Éléments } & NRD & NME & $\%$ survie & $\%$ os complets & NRDcut \\
\hline Pubis & PU & 0 & - & - & - & - \\
\hline Fémur & FE & 55 & 6 & $50,0 \%$ & 0,0 & 12 \\
\hline Proximal & FE1 & 10 & 6 & $50,0 \%$ & 0,0 & \\
\hline Diaphyse & FE2 & 31 & 5 & $41,7 \%$ & 0,0 & \\
\hline Distal & FE3 & 14 & 5 & $41,7 \%$ & 0,0 & \\
\hline Tibia & TI & 36 & 4 & $33,3 \%$ & 0,0 & 18 \\
\hline Proximal & TI1 & 9 & 4 & $33,3 \%$ & 0,0 & \\
\hline Diaphyse & TI2 & 24 & 4 & $33,3 \%$ & 0,0 & \\
\hline Distal & TI3 & 3 & 3 & $25,0 \%$ & 0,0 & \\
\hline Fibula & Fib. & 1 & 1 & $8,3 \%$ & 0,0 & 0 \\
\hline Patella & PA & 0 & - & - & - & - \\
\hline Calcaneus & $\mathrm{CA}$ & 6 & 2 & $16,7 \%$ & 16,7 & 2 \\
\hline Talus & AS & 2 & 2 & $16,7 \%$ & 50,0 & 0 \\
\hline Naviculaire & Nav. & 2 & 2 & $16,7 \%$ & 50,0 & 0 \\
\hline Grand Cunéiforme & Gcun. & 7 & 4 & $33,3 \%$ & 14,3 & 0 \\
\hline Petit Cunéïforme & Pcun. & 1 & 1 & $8,3 \%$ & 100,0 & 1 \\
\hline Cuboïde & Cub. & 3 & 3 & $25,0 \%$ & 33,3 & 1 \\
\hline Scaphoïde/Grand cunéïforme & - & 1 & - & - & 0,0 & 0 \\
\hline Métatarsien 3 & MT & 30 & 8 & $66,7 \%$ & 0,0 & 15 \\
\hline Proximal & MT1 & 24 & & $66,7 \%$ & 20,8 & \\
\hline Diaphyse & MT2 & 18 & & $41,7 \%$ & 0,0 & \\
\hline Distal & MT3 & 8 & & $58,3 \%$ & 62,5 & \\
\hline Métatarsiens accessoires & MTacc & 14 & 8 & $33,3 \%$ & 0,0 & 6 \\
\hline Phalanges 1 & $\mathrm{P} 1$ & 11 & 3 & $12,5 \%$ & 18,2 & 8 \\
\hline Phalanges 2 & $\mathrm{P} 2$ & 10 & 1 & $4,2 \%$ & 30,0 & 4 \\
\hline Phalanges 3 & P3 & 7 & 1 & $4,2 \%$ & 0,0 & 0 \\
\hline Grand sésamoïde & - & 7 & 7 & $14,6 \%$ & 100,0 & 0 \\
\hline Petit sésamoïde & - & 1 & 1 & $4,2 \%$ & 100,0 & 0 \\
\hline Métapodes & MP & 6 & - & - & 0,0 & 1 \\
\hline Métapodes accessoires & MPacc & 5 & - & - & 0,0 & 0 \\
\hline Total & & 853 & - & - & 13,6 & 193 \\
\hline
\end{tabular}

Tabl. 5 (suite et fin) - Abri Faustin. Nombre de restes déterminés (NRD), nombre minimal d'éléments (NME), pourcentages de survie et nombre de restes déterminés portant des stries de découpe (NRDcut) pour chaque élément squelettique de cheval de la travée 24.

Table 5 (end) - Abri Faustin. Number of identified specimens (NISP), minimum number of elements (MNE), survival-percentage rates, and number of identified specimens displaying cut marks (NISPcut) for each horse skeletal element in sector 24.

mités articulaires des os longs sont très rarement complètes, exception faite des métapodiens. Toutefois, cette fragmentation n'empêche pas leur identification puisque les pièces sont généralement supérieures à $3 \mathrm{~cm}$.

\section{Remontages}

Au total, 78 remontages osseux et dentaires ont été effectués sur la travée 24 , dont $65(83,3 \%)$ sur des restes provenant d'un même carré. Un métacarpien est entièrement remonté à partir de fragments provenant tous du carré L24 et présentant des cassures sur os frais (fig. 4). Sur la travée 25 , au moins une série dentaire a été retrouvée en place. Ces résultats vont dans le sens d'une faible dispersion des restes après leur abandon.

\section{Représentation osseuse}

Toutes les parties du squelette sont identifiées (tabl. 5). Toutefois, les éléments anatomiques sont présents en proportions très inégales. Les éléments les mieux conservés sont les os longs, les métapodiens et la mandibule tandis que l'axis, le sacrum, la scapula, la patella, l'os pétreux et l'hamatum sont absents. La relation statistique entre le pourcentage de survie et la densité osseuse n'est pas significative $(\mathrm{Rs}=0,12 ; \mathrm{p}=0,4$; fig. 5). Ainsi, certaines 
portions squelettiques à faible densité sont bien représentées (proximale et distale du fémur, proximale du tibia, proximale et distale de métatarsien) comparativement à d'autres dont la densité est beaucoup plus élevée (pétreux, acétabulum, ilium). Parallèlement, nous avons déterminé plusieurs restes de faible densité comme ceux de fotus et de cartilages costaux.

L'analyse de la représentation squelettique en fonction des indices d'utilité nutritive en viande (fig. 6) n'a montré aucune corrélation statistiquement significative (FUI : Rs $=-0,18 ; p=0,47$ ). Les éléments les mieux représentés dans l'assemblage sont en effet ceux dont les valeurs de FUI sont parmi les plus faibles (troisième métatarsien, mandibule). A contrario, les vertèbres cervicales et thoraciques ainsi que le pelvis, dont les valeurs de FUI sont fortes, montrent des pourcentages de survie très faibles. L'analyse statistique ne met également pas en évidence de corrélation entre l'indice d'utilité nutritive en moelle et la représentation osseuse (fig. 7 ; MUI : Rs = $0.40 ; p=0,33)$. Cette absence de relation entre les deux variables est due principalement à l'abondance du canon postérieur et de la mandibule.
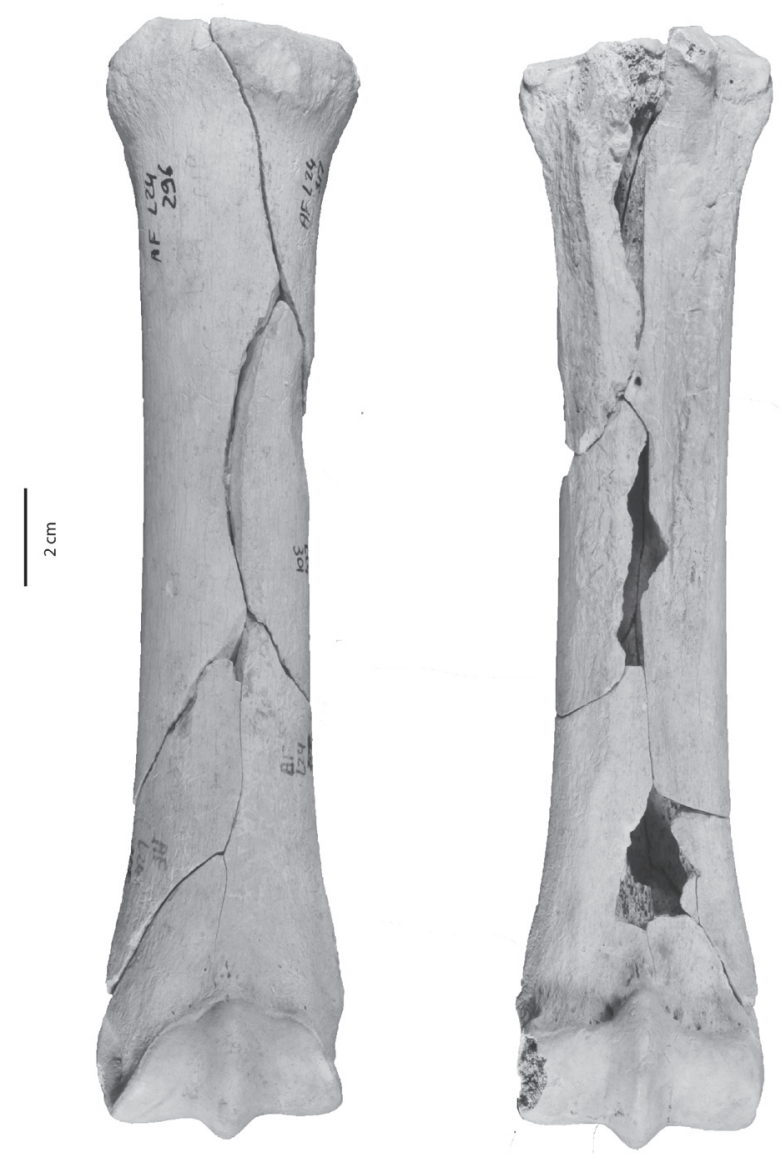

Fig. 4 - Abri Faustin. Remontage d'un métacarpien droit de cheval fracturé intentionnellement (vue antérieure à gauche; vue postérieure à droite). $\mathrm{NR}=5$.

Fig. 4-Abri Faustin. Reassembly of an intentionally-fractured right metacarpal of a horse (anterior view on the left; posterior view on the right). NSP $=5$.

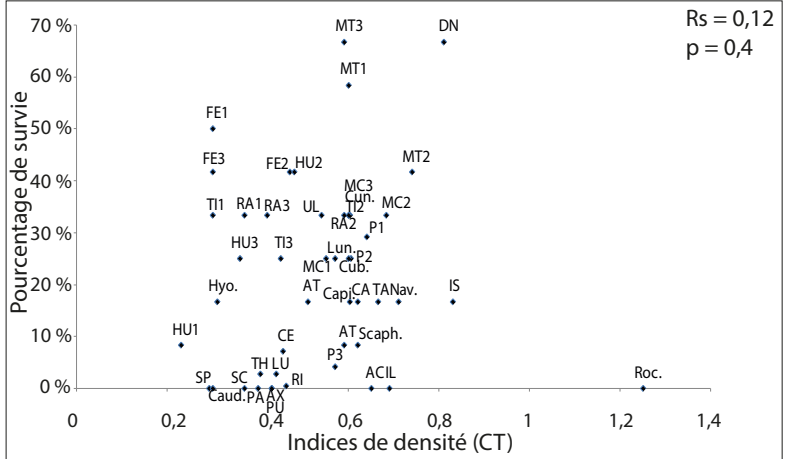

Fig. 5 - Abri Faustin. Diagramme de dispersion des pourcentages de survie en fonction de la densité des éléments (CT). Les abréviations utilisées sont explicitées dans le tableau 5.

Fig. 5 - Abri Faustin. Scatter diagram of percent-survival rates according to the density of the elements (CT). Abbreviations are explained in table 5.

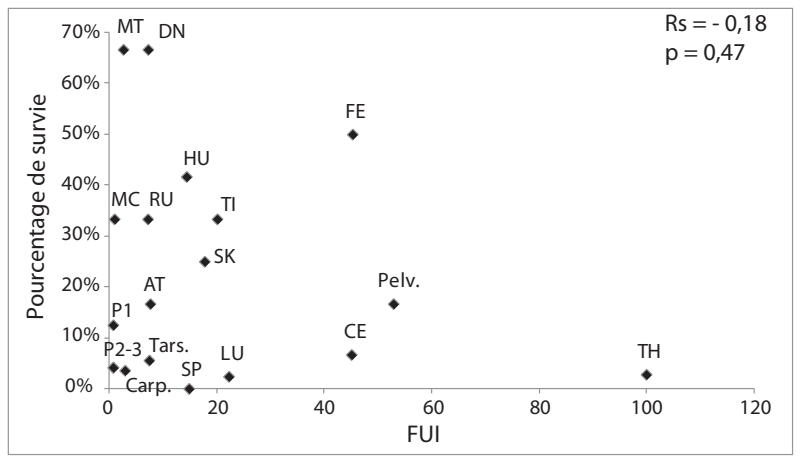

Fig. 6 - Abri Faustin. Diagramme de dispersion des pourcentages de survie en fonction du Food Utility Indice (FUI). Les abréviations utilisées sont explicitées dans le tableau 5.

Fig. 6-Abri Faustin. Scatter diagram of percent-survival rates according to the Food Utility Index (FUI). Abbreviations are explained in table 5.

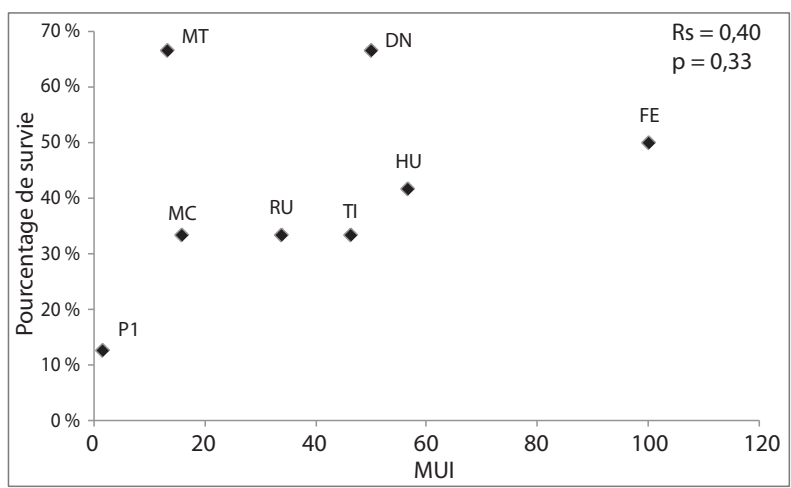

Fig. 7 - Abri Faustin. Diagramme de dispersion des pourcentages de survie en fonction du Marrow Utility Indice (MUI). Les abréviations utilisées sont explicitées dans le tableau 5.

Fig. 7 - Abri Faustin. Scatter diagram of percent-survival rates according to the Marrow Utility Index (MUI). Abbreviations are explained in table 5. 


\section{Populations chassées}

\section{Structures d'âges}

Le remontage des séries dentaires permet de réaliser une première estimation du nombre minimal d'individus en ne tenant compte que de la maturation dentaire, de l'usure et de la latéralisation (fig. 8). Sur la base de ces critères, il nous a été possible de décompter :

- cinq individus juvéniles (A1 à A5) et huit adultes (A6 à A13) et deux vieux adultes à partir des jugales inférieures droites;

- quatre individus (B1 à B4) juvéniles, deux subadultes (B5 et B7), deux adultes (B6 et B8), deux vieux adultes (B9 et B10) et un individu adulte d'âge indéterminé à partir des jugales inférieures gauches ;

- deux individus juvéniles (D1 et D2 ${ }^{(1)}$ ), deux subadultes (D3 à D4), sept adultes (D5 à D11), deux vieux adultes (D12 à D13) et un très vieux adulte (D14) à partir des jugales supérieures droites;

- deux individus juvéniles $\left(\mathrm{C} 1\right.$ à $\left.\mathrm{C} 4{ }^{(2)}\right)$, deux subadultes ( $\mathrm{C} 5$ et $\mathrm{C} 6)$, deux adultes ( $\mathrm{C} 7$ à $\mathrm{C} 8)$, deux très vieux adultes $(\mathrm{C} 9$ à $\mathrm{C} 10)$ à partir des jugales supérieures gauches.

La population de chevaux à Faustin est ainsi estimée à quinze individus sur la base des mâchelières des hémimandibules droites (NMI de fréquence; NMIf). Si l'on calcule un NMI de combinaison (NMIc) sur la base des classes d'âges attribuées aux différentes séries dentaires, le nombre minimal de chevaux abattus s'élève à dix-neuf.

Le profil d'abattage de la population équine de Faustin a été établi à partir de la moyenne des âges obtenus pour les dents de chaque série. Afin de limiter les imprécisions inhérentes à la méthode retenue, les résultats ont été répartis en cinq classes d'âges (fig. 9). Toutes les classes d'âges sont représentées. Toutefois, une fois le nombre d'individus rapporté au nombre d'années correspondant à chaque classe d'âge, il apparaît que les individus de moins de 2 ans sont les plus représentés, suivis par les individus âgés de 5 à 10 ans. Les sub-adultes et les vieux et très vieux individus sont les moins nombreux.

Si l'on se réfère aux modèles établis par Levine (1983), ce type de profil pourrait se rapprocher de pratiques de chasse opérées sur les groupes familiaux où les poulains et les femelles adultes dans la force de l'âge sont majoritaires et où les sub-adultes sont faiblement représentés en raison du départ des mâles vers les groupes de célibataires.

Sexe

La présence de plusieurs canines, correspondant à au moins deux individus, peut témoigner de l'abattage d'un maximum de deux mâles dans l'échantillon. L'une appartient sans nul doute à un jeune individu et la seconde, très usée, à un très vieil individu. Les groupes familiaux ne sont généralement composés que d'un seul mâle (l'étalon). Ce dernier peut, dans de très rares cas, tolérer la présence d'un second mâle, généralement âgé (Bignon,
2003). Il faut toutefois signaler que $20 \%$ des femelles de chevaux domestiques ont des canines (Barone, 1986). Par conséquent, ces deux individus peuvent aussi bien être des femelles que des mâles abattus lors d'épisodes de chasse sur groupes familiaux, ou de célibataires.

\section{Saisonnalité}

Les indices de saisonnalité sont issus de l'étude des dents jugales déciduales d'une part, et des restes fœtaux d'autre part. Les âges dentaires calculés pour les quinze séries déciduales se répartissent entre 6 et 22 mois (fig. 8). Sachant que les naissances se répartissent d'avril à juin avec un pic en mai, le mois d'abattage peut être connu avec un intervalle de confiance de plus ou moins un mois (fig. 10). Ainsi, à Faustin, il apparaît que les poulains ont été abattus tout au long de l'année avec cependant une prédominance entre les mois de janvier et de mai.

Les restes de foetus ont, quant à eux, fourni un âge calendaire se répartissant entre 15 et 20 semaines. La période de rut ayant lieu 15 jours après la mise bas, ces juments ont été abattues entre la fin de l'été et le début de l'hiver (fig. 10). Ces résultats suggèrent un abattage des chevaux, au sein des groupes familiaux, durant toute l'année avec, semble-t-il, une légère accentuation entre l'hiver et le printemps.

\section{Boucherie}

Le rapport NRDcut/NRD est de $22,6 \%$ pour la totalité des restes de chevaux (33,3\% dents exclues) et de $32,7 \%$ (44,5\% dents exclues) si l'on ne considère que les restes dont la surface est bien lisible (plus de $50 \%$ de la surface). Une fréquence élevée des stries de découpe, comme c'est le cas ici, est généralement synonyme d'un impact anthropique important.

\section{Crâne}

La fréquence des stries sur le crâne est très en-deçà de la moyenne pour l'assemblage (12,5\%). Sur les onze fragments portant des stries, neuf sont localisés anatomiquement de manière précise. Le maxillaire, l'os palatin et le basisphénoïde sont les seuls os du crâne portant des stries de découpe. Sur le maxillaire, elles se situent au niveau du tubercule facial et sur le bord alvéolaire. Ce type de stries est généralement associé au dépouillement de l'animal. Les deux fragments de basisphénoïde identifiés et deux fragments d'os palatin portent, quant à eux, des stries se rapportant de toute évidence au prélèvement de la langue.

\section{Mandibule (fig. 11a)}

Sur les cent vingt-deux fragments de mandibule, trente-cinq $(28,5 \%)$ portent des stries de découpe, dont vingt-quatre ont pu être replacées anatomiquement. Les stries situées sur la surface inférieure de la symphyse mandibulaire sont des témoignages du dépouillement (Binford, 


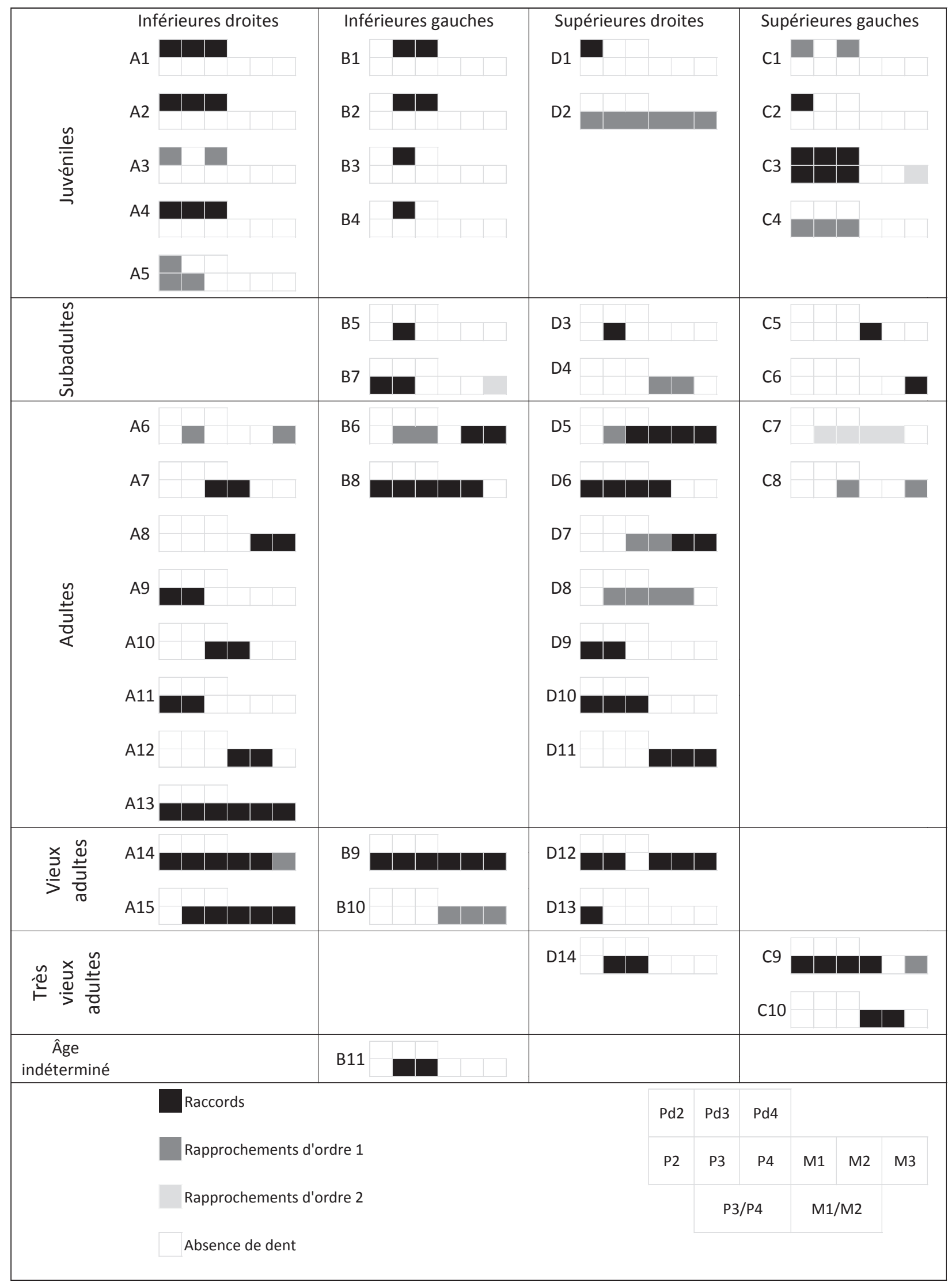

Fig. 8 - Abri Faustin. Répartition des dents des travées 24 et 25 par série. Les séries sont rangées de haut en bas dans l'ordre croissant de maturation dentaire.

Fig. 8 -Abri Faustin. Distribution of teeth in sectors 24 and 25 by series. The series are arranged from top to bottom in ascending order of dental maturity. 


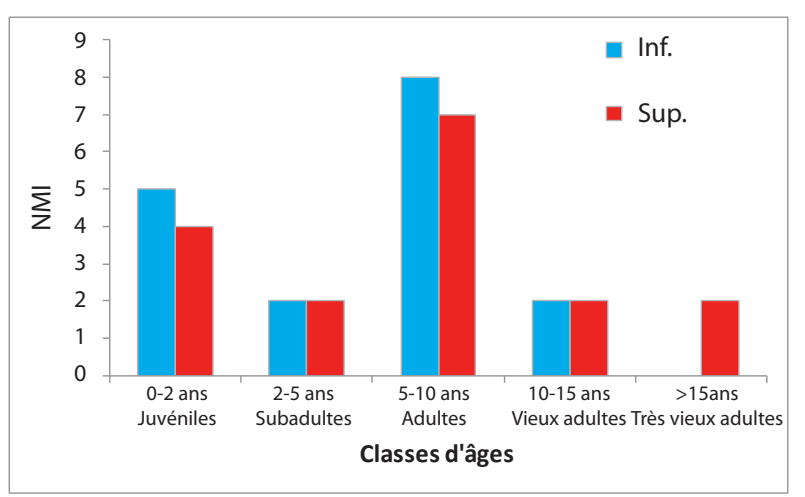

Fig. 9 - Abri Faustin. Profil de mortalité des chevaux de l'abri Faustin.

Fig. 9 - Abri Faustin. Horse mortality profile at Abri Faustin.

1981). Il en va de même pour les stries courtes sur le bord de la mandibule. L'incisure mandibulaire, la branche verticale de la mandibule et le processus coronoïde présentent des stries produites par la désarticulation de la mandibule (Nilssen, 2000). Celles situées longitudinalement sur la face linguale de la partie molaire s'expliquent par le prélèvement de la langue (Binford, 1981; Nilssen, 2000; Morel et Müller, 1997). Les traces présentent au niveau de la fosse massétérique sont le fait de la décarnisation.
La moelle contenue dans la mandibule se trouve au niveau de la branche horizontale. Seuls deux fragments de cet élément portent des impacts de percussion rappelant le procédé décrit par Binford (1978), à savoir une fracturation de la mandibule à la base de la rangée dentaire conduisant à la fracturation des dents jugales (voir Morel et Müller, 1997). À Faustin, de nombreuses dents jugales inférieures sont fracturées de manière transversale (fig. 12). L'ensemble de ces indices témoigne d'une fréquente fracturation des mandibules par les Magdaléniens.

\section{Vertèbres}

L'atlas porte des stries attribuables à la désarticulation de la tête de l'animal (Binford, 1981; Nilssen, 2000). Les stries sur les processus épineux de deux vertèbres thoraciques résultent du prélèvement des filets (Binford, 1981). Un processus articulaire porte des stries attestant du détachement des côtes (Nilssen, 2000).

\section{Côtes (fig. 11b et 11c)}

Sur les trente-deux fragments de côtes portant des stries (46,4\% des fragments de côtes), seulement seize ont pu être précisément replacés anatomiquement. Les stries situées sur et à proximité du col de la tête des côtes

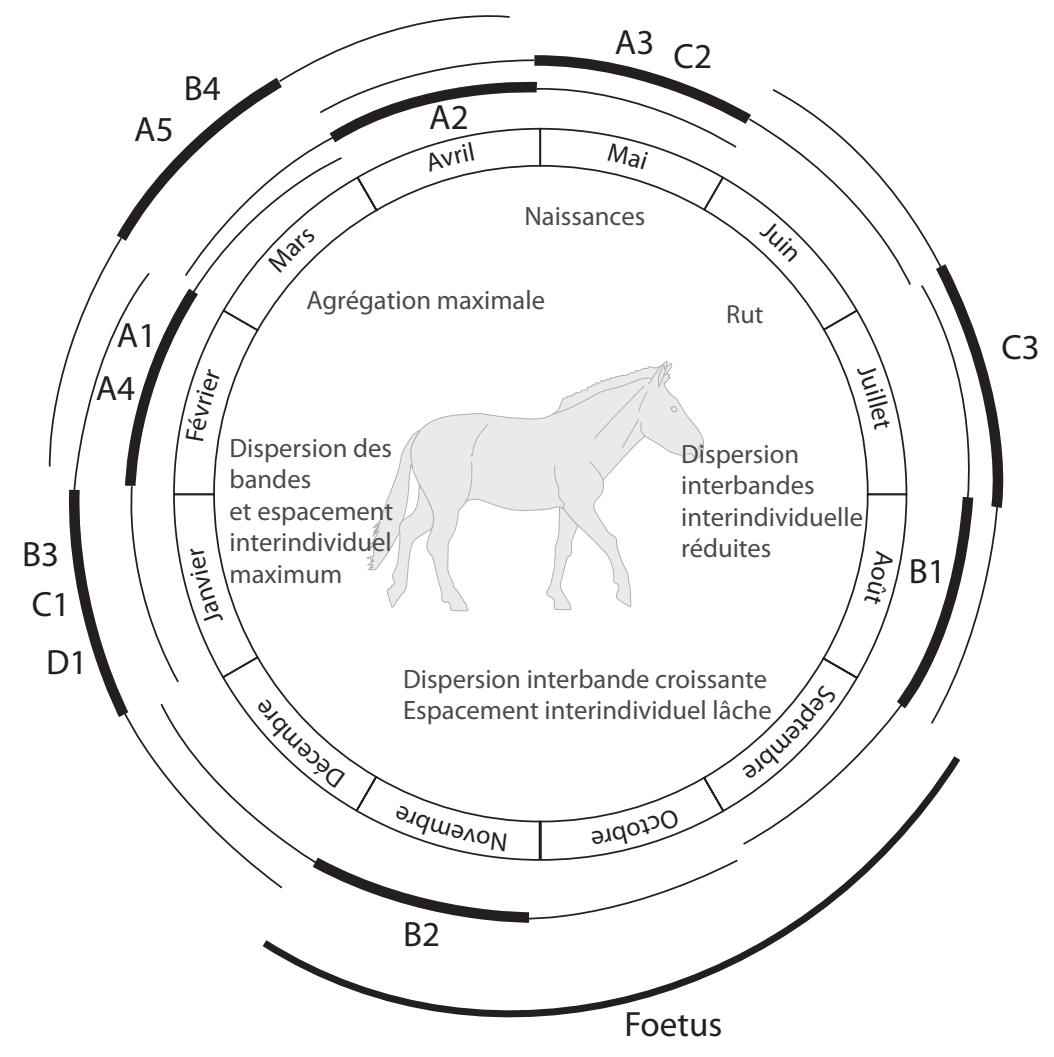

Fig. 10 - Abri Faustin. Estimation des mois de décès et des intervalles de confiance associés à partir des séries dentaires déciduales et des restes de fotus de cheval.

Fig. 10 - Abri Faustin. Estimation of month of death and associated confidence intervals based on deciduous dental series and horse foetus remains. 

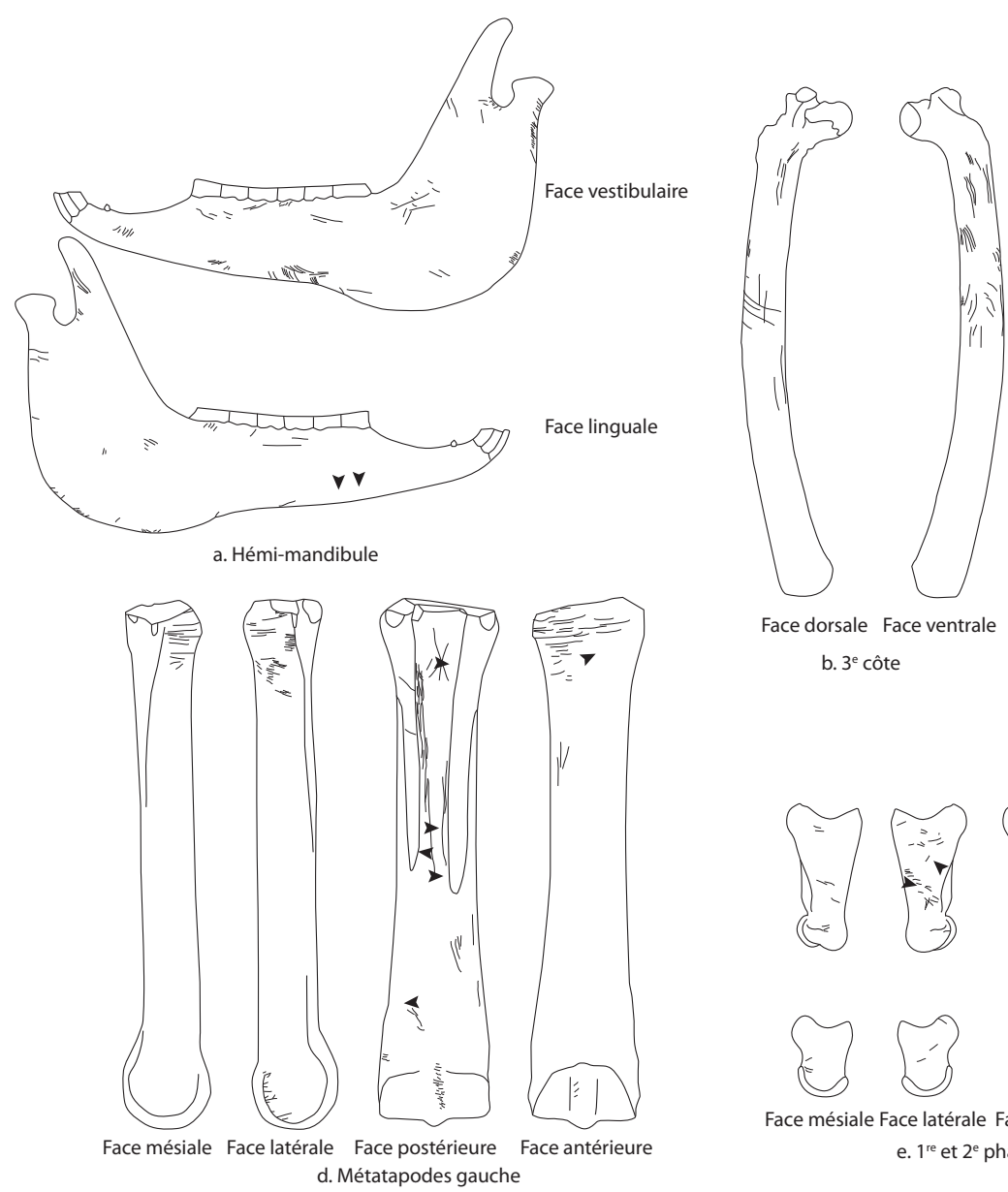

Face dorsale Face ventrale b. $3^{\mathrm{e}}$ côte
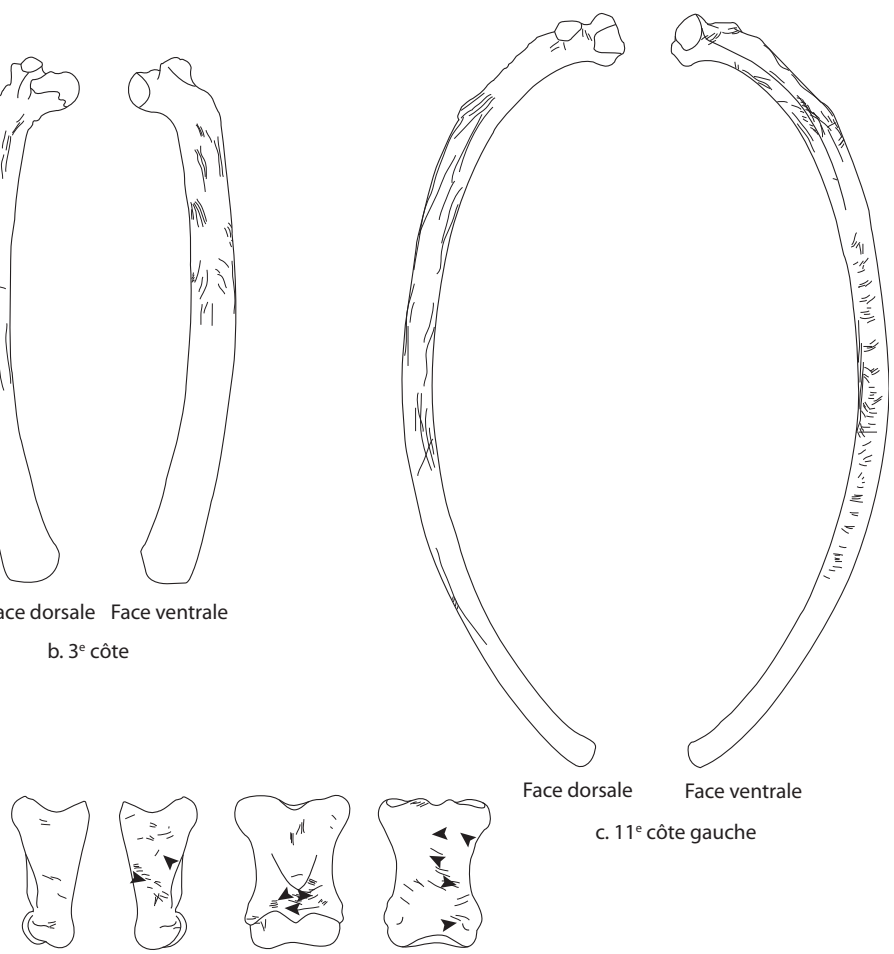

Face dorsale

c. $11^{\mathrm{e}}$ côte gauche

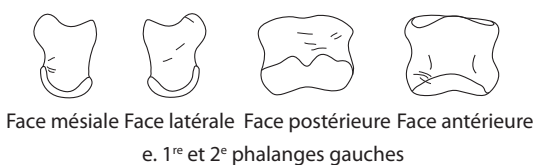

Fig. 11 - Abri Faustin. Localisation des stries et des impacts de percussion (flèches) sur la mandibule, les côtes, les métapodes et les phalanges de cheval.

Fig. 11 - Abri Faustin. Location of the cut marks and impact notches (arrow) oon horse mandible, ribs, metapodials, and phalanges.

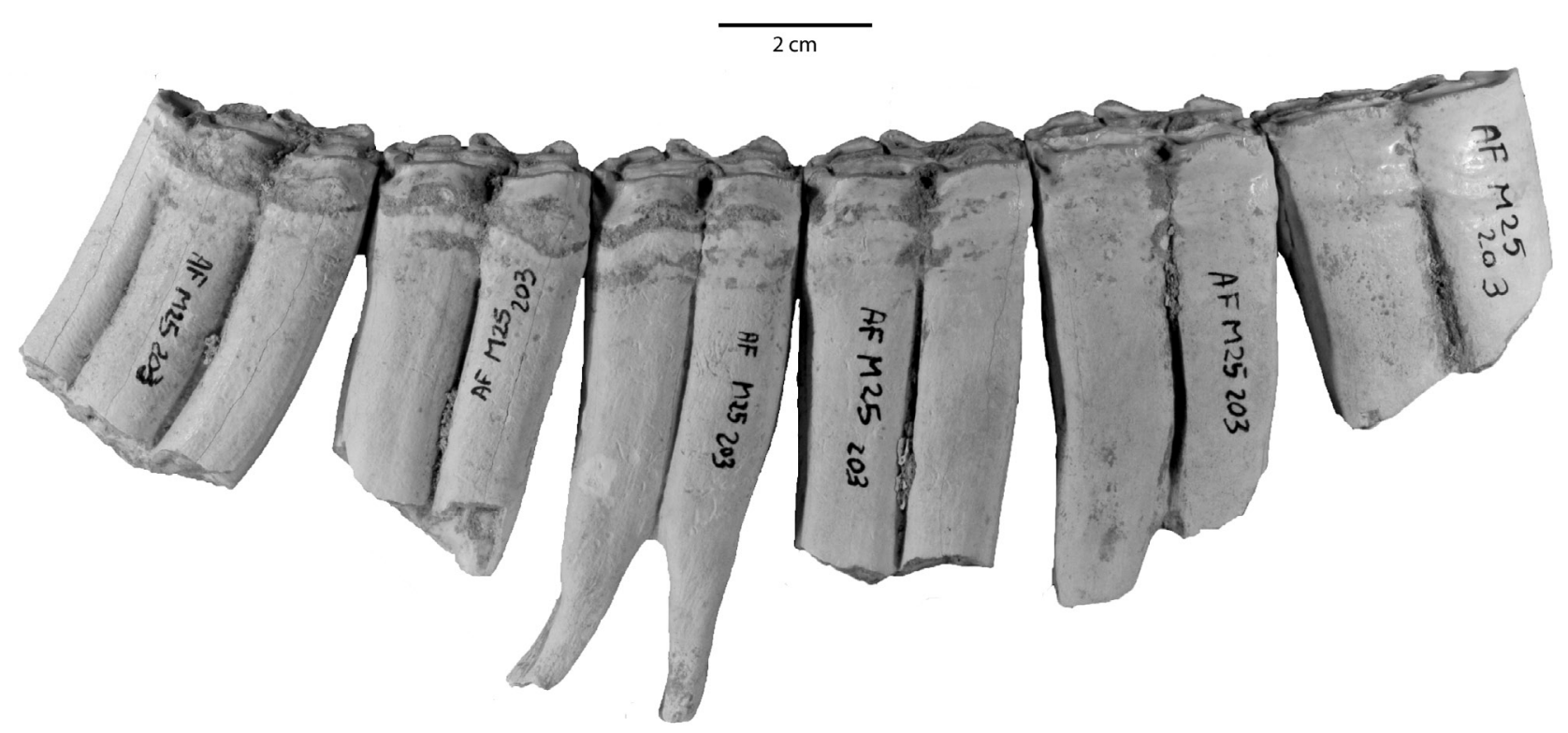

Fig. 12 - Abri Faustin. Série dentaire inférieure droite de cheval (Equus caballus) entièrement remontée en vue vestibulaire.

Fig. 12 - Abri Faustin. Lower right dental series for a horse (Equus caballus) entirely reassembled as a buccal view. 
témoignent de la désarticulation avec les vertèbres (voir supra). Les premières côtes ne présentent pas ce type de traces. Le corps des côtes porte des stries témoignant de deux activités distinctes. Celles sur la face médiale sont le résultat de l'éviscération; toutes les autres relèvent de la décarnisation et du prélèvement des filets (ibid.).

\section{Humérus (fig. 13a)}

L'humérus est l'os ayant la plus grande fréquence de stries $(65 \%)$. Elles se retrouvent en majorité sur la diaphyse au niveau des nombreuses insertions musculaires que possède cet os et sont, par conséquent, imputables à de la décarnisation (Binford, 1981; Nilssen, 2000). L'absence de traces de désarticulation au niveau de la diaphyse proximale est vraisemblablement le résultat de la rareté des fragments identifiés dans cette portion $(\mathrm{NME}=1)$. En revanche, quelques stries obliques sur la face médiale de la diaphyse, en partie proximale, sont le résultat de cette activité (Nilssen, 2000). La désarticulation du coude est documentée par des stries sur la face médiale de l'extrémité et une autre au niveau de la trochlée, sous la fosse coronoïdienne (Nilssen, 2000).
L'humérus est l'élément portant le plus d'impacts de percussion. La moitié d'entre eux sont situés sur la face antérieure de la diaphyse. Le reste des traces se répartit équitablement sur les autres faces. Enfin, des traces de chauffe ont été identifiées sur une extrémité distale d'humérus entière.

\section{Radio-ulnaire (fig. 13b)}

Le radio-ulnaire ne témoigne d'aucune trace relevant de la désarticulation. Les fragments d'extrémités articulaires sont pourtant bien représentés pour cet os $(\mathrm{NME}=4$ en proximal et distal). Ainsi, seul l'humérus porte de rares incisions attribuables à la désarticulation du coude. Cela signifie que, soit cette opération a été réalisée de manière très délicate, soit elle n'a pas été systématique (Müller, 2014). Les traces de découpe présentes sur la face médiale, les bords crânial et caudal de l'olécrâne, ainsi que sur la diaphyse du radius sont toutes associées à la décarnisation (Binford, 1981; Nilssen, 2000).

Les traces de percussion du radio-ulnaire sont toutes réparties sur les faces postérieure ou latérale. La répartition des impacts, alignés dans le sens de l'allongement de la diaphyse, suggère que les radius ont été fendus en long.
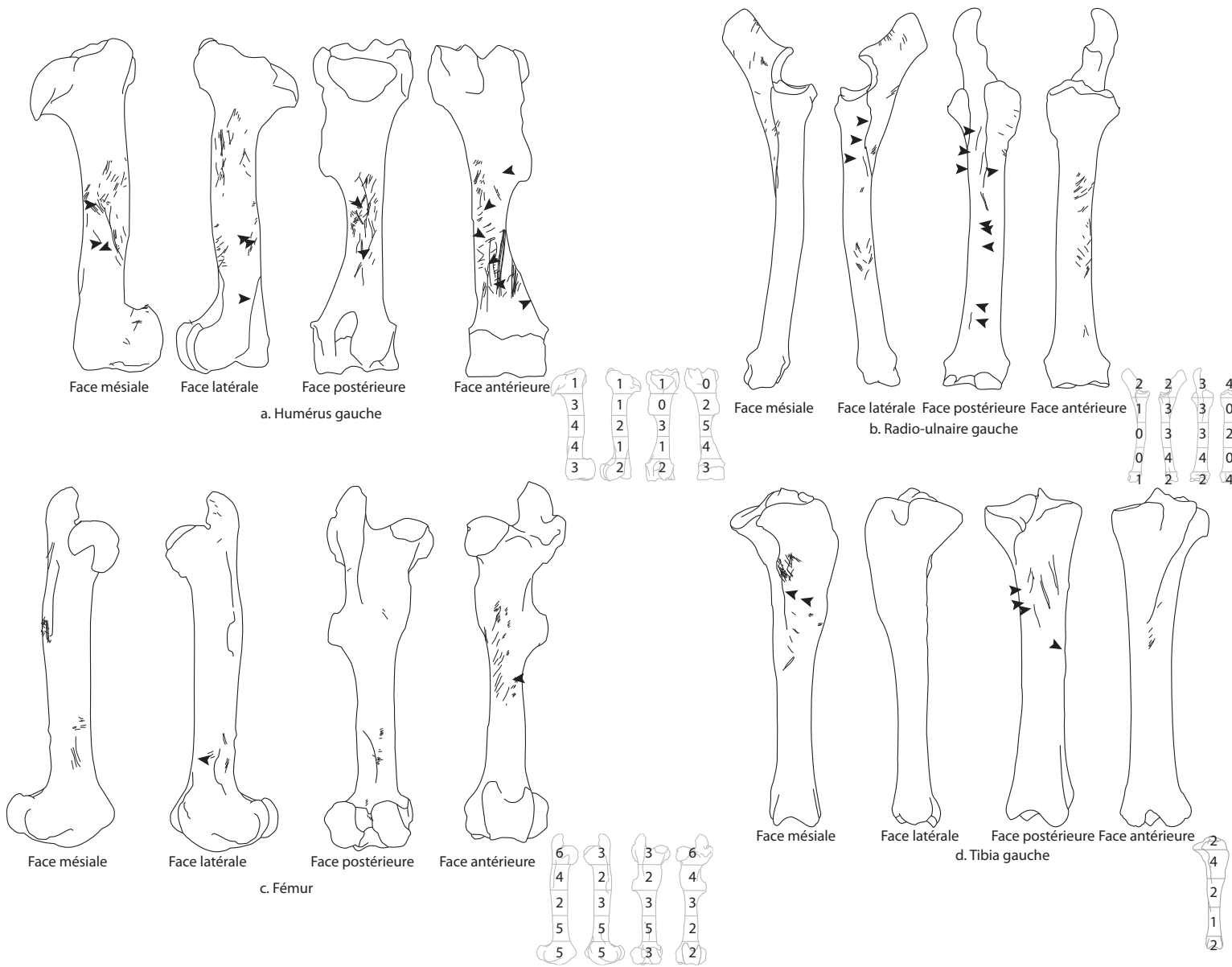

$\begin{array}{llll}0 & 3 & 3 & 2\end{array}$

$\begin{array}{llll}0 & 4 & 4 & 0\end{array}$

$\begin{array}{llll}0 & 4 & 4 & 0 \\ 1 & 2 & 2 & 4\end{array}$
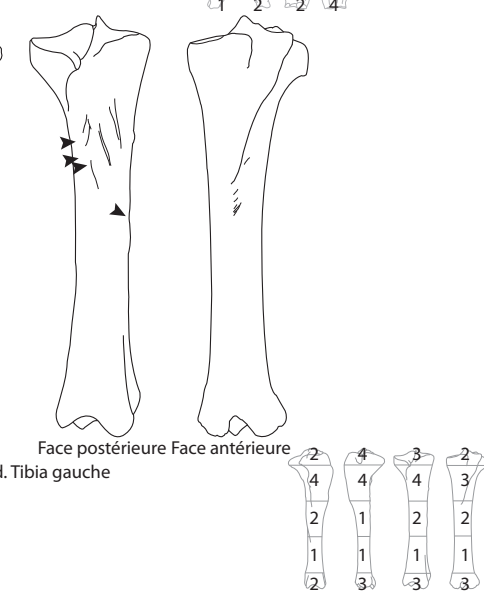

Fig. 13 - Abri Faustin. Localisation des stries et des impacts de percussion sur l'humérus, le radio-ulnaire, le fémur et le tibia. NME par portion anatomique.

Fig. 13 - Abri Faustin. Location of cut marks and impact notches on humerus, radio-ulnar, femur, and tibia. MNE by anatomical unit. 
Carpe

Les deux seuls os sur lesquels se trouvent des stries sont un capitatum et un triquetrum (tabl. 5). Elles s'expliquent par la désarticulation de l'avant-bras et du canon.

\section{Fémur (fig. 13c)}

Les extrémités articulaires du fémur, pourtant relativement nombreuses $(\mathrm{NME}=6$ en proximal; $\mathrm{NME}=5$ en distal), présentent très peu de traces de découpe. Deux fragments présentent des stries sur le grand trochanter. Elles ont pu se produire lors de la désarticulation avec le coxal (Binford, 1981). Un seul fragment d'extrémité articulaire distale a fourni des stries, situées dans la fosse inter-condylaire et peuvent correspondre à la désarticulation du fémur et du tibia. La rareté de ce type de traces, malgré le nombre important de ces éléments, peut signifier que ces articulations n'ont pas été systématiquement séparées. Une autre hypothèse suppose que la désarticulation de la hanche a pu être produite par torsion (Müller, 2014) mais nous ne disposons d'aucun autre élément permettant de l'étayer. Par ailleurs, Müller (2014) signale que la séparation du fémur et du tibia est aisée sans laisser de traces sur ces os. Toutes les stries présentes sur la diaphyse (crête trochantérique, tubérosité du muscle biceps, petit trochanter, face antérieure, tubérosité supracondylaire médiale et latérale) sont le fait de la décarnisation (Binford, 1981; Nilssen, 2000; Müller, 2014).

Deux traces de percussion ont été identifiées sur le fémur. Elles s'observent au milieu de la diaphyse, sur la face antérieure, et à la base, sur la face latérale. Leur faible quantité ne permet pas de mettre en évidence un schéma récurrent de fracturation de cet élément.

\section{Tibia (fig. 13d)}

Aucune strie n'a été relevée sur les extrémités articulaires du tibia. Ce fait pourrait être en lien avec une relative rareté des fragments puisque les NME calculés pour ces portions sont de quatre et trois respectivement en partie proximale et en partie distale. Mais également, comme nous l'avons vu, la désarticulation du genou a pu se faire facilement en ne laissant aucune trace. Ainsi, cet os n'a fourni que des stries relevant de la décarnisation. La rareté des traces de découpe en partie distale de la diaphyse est liée d'une part à la rareté des fragments pour cette portion $(\mathrm{NME}=2)$ et d'autre part à l'absence d'insertion musculaire dans cette zone.

Le tibia porte également peu de traces de percussion. Elles sont toutes situées dans la partie proximale de la diaphyse, sur les arêtes latérale et mésiale.

\section{Tarse}

Tous les os formant le tarse ont été identifiés. Seuls deux calcaneus, un complet (le seul) et un fragment proximal, un petit cunéiforme, et un cuboïde portent des traces de boucherie (tabl. 5). Ces stries se rapportent toutes à de la désarticulation (Binford, 1981). Celles sur le calcaneus sont relatives à la séparation du tibia et du tarse, les autres à la dislocation entre le tarse et le métatarse.

\section{Métapodiens (fig. 11d)}

Les métapodiens accessoires ne sont représentés que par les métatarsiens IV $(\mathrm{NRD}=8 ;$ NRDcut $=3)$ et II $(\mathrm{NRD}=4$; NRDcut $=1)$, la détermination des autres n'ayant pas pu être plus précises. Les stries identifiées sont transverses et situées aux extrémités proximales. Ce type de stries est relatif à la désarticulation entre le basipode et le métapode. Les faces antérieures des os canons portent également des traces de découpe transverses en partie proximale qui renvoient elles aussi au procédé de désarticulation précédemment décrit. Les stries longitudinales sur la face postérieure s'expliquent par l'extraction des tendons des muscles fléchisseurs (Müller, 2014). Enfin, les condyles présentent des stries courtes et transverses relatives à la désarticulation de la première phalange (Binford, 1981; Costamagno et David, 2009).

Une unique trace de percussion est identifiée sur la face antérieure et cinq sont situées le long de la diaphyse sur la face postérieure. Ces dernières appartiennent toutes à un seul métacarpe entièrement remonté (fig. 4). Ce métacarpe a été fendu en long, selon le procédé envisagé pour le radius.

\section{Phalanges (fig. 11e)}

Les premières phalanges montrent une fréquence des traces de boucherie relativement importantes avec huit restes sur onze. Un peu moins de la moitié des deuxièmes phalanges présentent des stries (tabl. 5). Les quelques fragments de troisième phalange sont trop fragmentés et trop mal conservés pour permettre l'observation d'éventuelles traces anthropiques. En revanche, le corps des premières et deuxièmes phalanges présente de nombreuses stries de dépouillement. La désarticulation entre les métapodes et les premières phalanges, entre les premières et deuxièmes et enfin entre les deuxièmes et troisièmes phalanges est documentée. Enfin, les stries en face postérieure des phalanges témoignent du prélèvement des tendons (Costamagno, comm. pers).

Deux premières phalanges ont été retrouvées complètes, les six autres ont été fendues en long et portent des impacts de percussion sur leurs faces antérieure et postérieure. Les deuxièmes phalanges ont presque toutes été trouvées complètes (3 sur 4). Les bords de cassure du seul fragment n'ont pas permis de mettre en évidence de fracturation.

\section{Exploitation des sous-produits}

Nous avons identifié un unique reste de métapode témoignant de l'utilisation de cet os pour l'extraction de baguette par rainurage longitudinal, probablement pour la confection d'une aiguille. Enfin, plusieurs os plats non déterminés spécifiquement mais appartenant à un ongulé de type $3 / 4$ portent des traits de gravures, et un fragment d'olécrâne d'ulna est recouvert d'ocre. 


\section{DISCUSSION}

\section{Taphonomie}

Nous avons vu précédemment que les carnivores, s'ils sont intervenus sur l'assemblage, n'ont pas laissé l'empreinte de leur passage. Quant aux rongeurs, leur action est négligeable. Les Magdaléniens apparaissent seuls responsables de l'accumulation. Le weathering, bien que touchant de nombreux vestiges, n'est que de faible intensité puisqu'il dépasse rarement le stade 1 . Pour autant, cela ne nous permet pas de conclure à un enfouissement rapide puisque des expérimentations ont montré qu'en milieu continental froid, les ossements peuvent rester intacts après une exposition de plusieurs années, en particulier s'ils sont protégés des ultraviolets solaires (Mallye et al., 2009; Bertran et al., 2015). Les phénomènes taphonomiques post-enfouissement sont ceux qui ont laissé la plus forte empreinte sur l'assemblage : les racines, la dissolution et les concrétions calcitiques. Ces deux dernières sont des indicateurs de circulation d'eau. Malgré cela, la conservation différentielle ne semble pas avoir eu d'impact significatif sur l'assemblage. Enfin, les nombreux remontages sur de courtes distances témoignent d'une dispersion limitée des vestiges. Ces résultats montrent que les processus post-abandon et la géodynamique du gisement n'ont pas eu d'impact majeur sur l'assemblage.

\section{Tactiques de chasses}

À Faustin, le cheval est sans conteste l'ongulé le plus abondant. Avec cinq poulains, dix adultes comprenant au moins deux femelles gestantes et deux mâles, les indices de saisonnalité montrent que les chevaux ont été chassés tout au long de l'année, même si l'hiver et le printemps ont pu être privilégiés. Cette période correspond à un moment d'affaiblissement des individus en raison de leur mauvaise condition physique, particulièrement à la sortie de l'hiver. D'autre part, leur faible mobilité au moment de la mise bas, au printemps, en fait des proies plus faciles. À l'inverse, les chevaux n'ont pas ou presque pas été chassés en début d'été (juin) et à la fin de l'automne (octobre à décembre). Les deux individus supposés mâles abattus qui ont été identifiés peuvent correspondre à des célibataires ou appartenir à des groupes familiaux.

La chasse préférentielle sur les groupes familiaux à l'abri Faustin peut s'expliquer par une plus grande prévisibilité des trajets et des comportements de ces groupes, associés à leur vulnérabilité plus importante en raison de la présence des poulains et de femelles gestantes. Les sentiers qu'ils laissent permettent de les repérer relativement facilement dans le paysage. Tandis que les groupes familiaux empruntent toujours les mêmes circuits, les déplacements des groupes de mâles célibataires sont plus erratiques (West, 1997) bien que ceux-ci aient tendance à tourner autour des premiers (Bignon, comm. pers.). Les déplacements des groupes sont rythmés par les espacements et regroupements des individus. En hiver, les chevaux - à la recherche de zones abritées - occupent des territoires vastes, l'espacement inter-individuel est alors maximal et ils sont plus difficiles à repérer dans le paysage. Du printemps à l'été, leur territoire est plus réduit et les deux types de groupes sont plus souvent en interaction en raison de la période de reproduction et des combats pour le contrôle des harems. Les chevaux occupent alors des espaces ouverts, leur permettant de repérer très tôt l'approche d'un danger. Durant cette période, ils sont certes plus faciles à localiser, mais aussi plus difficiles à approcher.

Si la chasse sur les groupes familiaux a pu être planifiée, il est fort probable que celle menée sur les groupes de célibataires ou les individus isolés ait été plus opportuniste. Le cheval sauvage étant très méfiant et ayant une distance de fuite de plusieurs kilomètres, il est très difficile de l'approcher en espace découvert. Toutefois, l'Entredeux-Mers étant une région vallonnée, les Magdaléniens ont pu savoir tirer profit de cet avantage topographique. Se pose alors la question de la tactique de chasse mise en œuvre. Le profil de mortalité montre une répartition des âges typique de l'abattage d'un groupe familial. Les chevaux ont donc été abattus sans critères de sélection d'âge. La technique décrite par Müller (2014) pour les chevaux de Monruz est tout à fait envisageable à Faustin. Les Magdaléniens se seraient postés à l'affût sur les circuits empruntés régulièrement par les hardes pour que la distance d'attaque soit la plus réduite possible. Il se peut que des techniques de rabattage aient été utilisées pour guider les chevaux en direction des chasseurs embusqués. Que les chevaux se soient dirigés eux-mêmes vers l'embuscade ou qu'ils y aient été guidés, les chasseurs ne disposaient que de très peu de temps une fois repérés avant que les chevaux ne prennent la fuite et se retrouvent rapidement hors d'atteinte. Devant viser les individus les plus proches, aucune sélection d'âge ne pouvait être établie. Cette technique devait permettre de tuer au plus deux à trois individus à chaque épisode de chasse (Müller, 2014). Ce chiffre pouvait évidemment varier en fonction du nombre de chasseurs placés en embuscade.

\section{Exploitation des carcasses}

De fortes disparités s'observent au niveau de la représentation osseuse. La densité osseuse et l'utilité nutritive en viande et en graisse ne sont pas corrélées à la représentation squelettique suggérant l'implication d'autres facteurs. Le déficit de certains éléments comme l'axis, le sacrum ou la scapula peut résulter d'un problème de détermination différentielle. La présence de 146 os plats classés en ongulé 3/4 (tabl. 6) étaye cette hypothèse. À l'inverse, l'abondance des fragments de métapodiens et de mandibules de chevaux, du fait de leur conformation, sont plus facilement déterminables. L'absence de certains éléments et la bonne représentation d'autres pourraient également résulter de l'emprise spatiale limitée de notre échantillon, un huitième seulement du site fouillé ayant été analysé.

La présence de toutes les parties squelettiques (tête, tronc, membres) dans l'assemblage nous incite à penser 


\begin{tabular}{|l|c|c|c|c|}
\multicolumn{1}{l|}{} & $\begin{array}{c}\text { Ongulés } \\
\text { indet. }\end{array}$ & $\begin{array}{c}\text { Ongulés } \\
2 / 3\end{array}$ & $\begin{array}{c}\text { Ongulés } \\
3 / 4\end{array}$ & Total \\
\hline Dents & 3 & 0 & 0 & 3 \\
\hline Os plats & 6 & 13 & 146 & 165 \\
\hline dont crâne & 3 & 0 & 34 & 37 \\
\hline dont côtes & 1 & 8 & 19 & 28 \\
\hline Vertèbres & 1 & 0 & 2 & 3 \\
\hline Cartillage caustal & 14 & 0 & 0 & 14 \\
\hline Os long & 6 & 4 & 81 & 91 \\
\hline dont humérus & 0 & 0 & 3 & 3 \\
\hline Os courts & 0 & 0 & 3 & 3 \\
\hline NID & 76 & 10 & 175 & 261 \\
\hline Total & 106 & 27 & 407 & 540 \\
\hline
\end{tabular}

Tabl. 6 - Abri Faustin. Décompte du nombre de restes selon les types d'ossement et par classes de taille d'ongulé pour la travée 24.

Table 6 - Abri Faustin. Breakdown of the number of specimens according to different bone types and ungulate size classes for sector 24.

que les carcasses ont été apportées entières sur le site. Toutefois, le contraste entre la bonne représentation des portions articulaires des os longs et le déficit du rachis pourrait suggérer une introduction incomplète de certaines carcasses, les filets étant retirés sur le site d'abattage. Néanmoins la bonne représentation du bloc crânio-facial, de l'atlas, de l'hyoïde, des côtes et des os longs nécessiterait une décapitation et un tronçonnement des plats de côtes et la désarticulation des membres avant le transport. La présence de restes fœtaux supposerait une éviscération sur le site d'abattage et le transport isolé de ces très jeunes sujets. Cette hypothèse d'un transport sélectif est certes plausible mais, selon nous, peu parcimonieuse du fait d'une longue préparation sur le site d'abattage.

Ainsi, si l'on considère un apport de carcasses complètes à Faustin, les restes de fœtus nous indiqueraient qu'au moins deux femelles gestantes ont été abattues et apportées entières à l'abri. Or, un cheval adulte pèse entre 250 et $300 \mathrm{~kg}$ (voir Discamps, 2011 pour une synthèse). Cela suggère que le site d'abattage se situait dans un périmètre restreint autour du gisement. On peut supposer que le choix de l'implantation de ce dernier est lié aux comportements des chevaux, les Magdaléniens ayant choisi un secteur régulièrement parcouru par les groupes, probablement une zone de recouvrement d'habitats de plusieurs bandes. En effet, lorsqu'un troupeau est chassé à un endroit donné, il aura tendance à éviter ce secteur pendant un moment. Il est donc peu plausible que les Magdaléniens aient chassé une seule harde.

\section{Exploitation alimentaire}

Les carcasses ont été entièrement traitées sur le gisement, après transport depuis le site d'abattage. Les stries de boucherie ont montré le prélèvement de toutes les masses carnées : muscles, langue et filets. L'abondance des stries de décharnement sur les ossements est généralement associée à un décharnement antérieur à la cuisson (Delpech et Villa, 1993; Costamagno et David, 2009; Soulier et al., 2014). Cela expliquerait l'extrême rareté des ossements portant des traces de chauffe ou de brûlure. En moyenne, la masse de viande fournie par une carcasse de cheval est évaluée à 146,5 kg (Outram et RowleyConwy, 1998). Ainsi, à Faustin, les quatorze individus adultes auraient fourni plus de deux tonnes de viande. Ce chiffre est néanmoins à relativiser en fonction notamment de la saison d'abattage.

La fracturation des os longs et de la mandibule, dans le but d'extraire la moelle et la graisse osseuse, est systématique à l'abri Faustin. Ce comportement semble être systématisé puisqu'un schéma répétitif dans le geste de fracturation a été relevé sur le radius, les métapodes consistant en un alignement de points d'impact sur leur face postérieure. Pour la mandibule, la fracturation transversale des dents jugales est un phénomène déjà observés au Grand Canton (Bridault et Bémili, 1999), à HauteriveChampréveyre et Monruz (Morel et Müller, 1997; Müller, 2014) et sur bien d'autres gisements. Elle est interprétée comme le résultat de la fracturation des mandibules pour la récupération de la moelle (Poplin, 1992 et 1994).

Si une grande partie de la moelle des os de chevaux peut être extraite facilement par fracturation, cela ne permet pas de l'extraire dans sa totalité. En effet, en raison du caractère très spongieux des cavités médullaires des os longs d'Équidés (Blumenschine et Madrigal, 1993; Lupo, 1998) une chaîne opératoire plus complexe est nécessaire. La confection de bouillons à l'aide de pierres chauffées pour l'obtention des matières grasses des os d'Équidés a été proposée (Lupo et Schmitt, 1997). Nous pensons que cette technique a été employée par les Magdaléniens de Faustin au regard des nombreuses pierres chauffées trouvées lors de la fouille (voir les carnets de fouille de M. Lenoir). Les extrémités articulaires des os longs ont par ailleurs été fracturées sans pour autant être broyées et brûlées. Dès lors, leur utilisation comme combustible ne peut être retenue. Ainsi, l'extraction de la graisse de ces portions et de la moelle des cavités médullaires pourrait suivre le même procédé opératoire réduisant de fait les coûts en temps, en combustible et en pierres que requiert cette technique.

Le poids de moelle pouvant être extraite sur une carcasse de cheval a été évalué par Outram et RowleyConwy (1998) à 227,3 g. Pour les quatorze individus adultes identifiés, cela correspondrait à plus de $3 \mathrm{~kg}$ de moelle. Ce calcul dépend encore une fois de la saison de chasse, mais également, comme nous l'avons vu, de la technique utilisée pour l'extraction. Celle-ci dépend certes des besoins du groupe mais également de sa rentabilité. En effet, la quantité de moelle fournie par un cheval est finalement extrêmement faible, surtout si elle est comparée à celle fournie par un renne (environ $6 \mathrm{~kg}$ selon Binford, 1978). Les besoins nutritionnels du groupe humain en graisse n'ont donc vraisemblablement pas pu être satisfaits uniquement par les apports lipidiques fournis par les chevaux. 


\section{Exploitation technique et symbolique}

Outre les besoins nutritionnels, l'exploitation des carcasses répond également à des besoins non alimentaires. On ne peut que supposer l'utilisation des matériaux périssables tels que les peaux, les tendons, les viscères. Le dépouillement et l'éviscération étant nécessaires au traitement des carcasses, les traces laissées par ces activités ne prouvent pas l'utilisation de ces matériaux après leur prélèvement. En revanche, le transport des carcasses entières, avant même l'éviscération pour au moins deux juments, suggère une volonté de récupérer au moins une partie des viscères ou de la peau des fœetus. Par ailleurs, les stries de dépouillement retrouvées sur les phalanges attestent du prélèvement de peaux fraîches de grandes tailles (Binford, 1981) par les Magdaléniens. Bien que les aiguilles aient pu servir à un simple entretien d'objets en peaux, leur présence pourrait indiquer une utilisation des peaux sur le gisement. Le temps nécessaire au séchage des peaux impliquerait alors une occupation longue du site bien que l'introduction de peaux déjà sèches soit une autre possibilité; une hypothèse n'excluant pas nécessairement l'autre. Peu d'indices attestent de l'utilisation des os de chevaux à Faustin pour la confection d'outils. Seul un métapodien ayant servi de matrice pour l'extraction d'éléments allongés, probablement pour la confection d'aiguilles, a été identifié.

Enfin, un fémur a servi de support pour une représentation de silhouettes féminines (Lenoir, 1983; Delporte, 1993).

\section{Fonction du site}

Le cheval est, sans conteste, l'espèce qui a fourni la plus grande quantité de ressources alimentaires à Faustin. Les dix-neuf chouettes harfang comptabilisées sur l'ensemble de la surface fouillée (Le Bail, 2005) ne représentent environ qu'un huitième du poids de viande fourni par un cheval. En revanche, l'exploitation alimentaire et, pourquoi pas, utilitaire de ce petit gibier, ainsi que du blaireau et du renard, témoigne d'une certaine diversité dans les activités pratiquées sur le site (Le Bail, 2005; Mallye, 2007; Val, 2009). Les industries lithiques et osseuses, se manifestant tant par l'outillage à vocation domestique que par de l'armement de chasse, ainsi que les éléments de parure et l'art mobilier identifié, reflètent également cette diversité d'activités (Lenoir, 1983) même si l'analyse fonctionnelle du matériel lithique et osseux demeure à faire. Par ailleurs, les données sur l'origine des matières premières lithiques vont dans le sens d'un approvisionnement de préférence en ressources locales (Lenoir, 1983) ${ }^{(3)}$. La même démarche semble avoir été adoptée pour les apports carnés, puisque plusieurs indicateurs tendent à démontrer que les gibiers ont été acquis à proximité du site.

Ces divers éléments sont des indices d'occupations longues et répétées de l'abri Faustin, qui a probablement fonctionné comme un camp résidentiel. Si l'on se réfère aux données sur la saisonnalité, uniquement fournies par le cheval, cette succession d'occupations s'est répartie tout au long de l'année, même si elles semblent avoir été plus sporadiques de l'été au tout début de l'hiver.

Ainsi, l'abri Faustin reflète parfaitement les transformations comportementales des chasseurs-cueilleurs du Magdalénien supérieur dans le Sud-Ouest de la France. Le tableau de chasse dominé pas les grands ongulés, notamment ici le cheval, se voit enrichi par des petits gibiers (Laroulandie, 2003 et 2004; Cochard, 2004; Cochard et Brugal, 2004; Costamagno et Laroulandie, 2004; Costamagno et al., 2008; Laroulandie, 2009) et un approvisionnement en ressources locales et régionales (Langlais et al., 2012 et 2014).

\section{DISCUSSION SUR L'EXPLOITATION DU CHEVAL AU MAGDALÉNIEN SUPÉRIEUR}

$\mathrm{D}^{\mathrm{c}}$ nombreux points de comparaisons peuvent être établis entre l'abri Faustin et les gisements contemporains du Magdalénien supérieur.

En premier lieu, à Faustin, comme dans le Bassin parisien et le plateau Suisse, l'abattage des chevaux s'est principalement concentré sur les groupes familiaux (Bignon, 2003; Morel et Müller, 1997; Müller, 2014). L'acquisition de chevaux provenant de groupes de célibataires reste, quant à elle, minoritaire. L'interception, à l'affût, des hardes de chevaux, avec ou sans rabattage, est la tactique de chasse généralement proposée pour les gisements où le cheval domine. C'est le cas à Solutré, Monruz, Hauterive-Champréveyres, et dans plusieurs gisements du Bassin parisien (Morel et Müller, 1997; Turner, 2002; Bignon, 2003; Müller, 2014). À Faustin, l'impossibilité de déceler des phases d'occupations distinctes ne permet pas de déterminer clairement si cette tactique de chasse a été adoptée, celle-ci ne pouvant qu'être supposée. Nos résultats sont donc globalement compatibles avec les stratégies décrites dans le Bassin parisien et sur le plateau Suisse.

Les données sur la saisonnalité ont montré que le cheval a été chassé toute l'année tant dans le Bassin parisien, que sur le plateau Suisse ou en Rhénanie centrale. (Morel et Müller, 1997; Bignon, 2003 : Street et al., 2006; Müller, 2014). C'est au printemps que les chasses sont majoritaires. L'automne, puis l'été sont également fortement représentés (Bignon, 2003). En revanche, les chasses hivernales se produisent essentiellement au début ou à la fin de cette saison. À Faustin, les chasses se sont également produites tout au long de l'année mais semblent avoir été plus fréquentes en hiver et au printemps; l'été, l'automne et le tout début de l'hiver étant moins bien représentés. Les raisons avancées pour expliquer la chasse des chevaux au cours de cette période sont d'ordre éthologique (voir supra). Ainsi, les différences dans la saison de chasse de cet Équidé peuvent s'expliquer par sa présence plus ou moins abondante saisonnièrement dans les différentes régions. Par ailleurs, il est important de souligner que les données saisonnières dans le Bassin parisien, en Rhénanie 
centrale et sur le plateau Suisse prennent en compte les études de plusieurs gisements. Or notre étude est centrée sur un unique gisement et les données saisonnières pour la chasse aux chevaux sur des gisements contemporains $d u$ nord de l'Aquitaine ne sont pas disponibles.

La taille et le poids des chevaux sont des facteurs déterminants pour les stratégies de transport mises en œuvre. Lorsque le cheval domine le spectre faunique, il fait rarement l'objet de transport différentiel. Dans le Bassin parisien, les chevaux semblent avoir été transportés du site d'abattage vers le site de traitement dans leur intégralité, suggérant une proximité entre ces deux lieux. Toutefois, les parties les plus riches sont parfois emportées ultérieurement (Bignon, 2003). Sur les sites de Hauterive-Champréveyres et Monruz, les carcasses ont été directement et intégralement traitées sur le lieu d'abattage (modèle « Camp to Big Game » de Morel et Müller, 1997; Müller, 2014). À Faustin, le transport des carcasses suggère, là aussi, que les Magdaléniens ont privilégié la proximité du lieu d'abattage et du gisement. Même si un débitage grossier a pu avoir lieu avant le transport, tous les éléments ont été apportés sans sélection en fonction de leur qualité nutritive.

La chaîne opératoire de boucherie ne montre pas de variation majeure entre les gisements et le prélèvement des produits alimentaires sur les carcasses est toujours complet. Seul Solutré fait exception puisqu'une grande partie des chevaux n'a pas été traitée et les portions squelettiques ne semblent pas avoir fait l'objet d'un transport secondaire (Olsen, 1989; Turner, 2002). Il faut envisager que sur ce dernier gisement les chevaux ont pu être abattus pour des raisons autres qu'alimentaires (Costamagno, 2003). Bien que l'utilisation des os de cheval à des fins techniques ne soit pas systématique, elle se retrouve de façon sporadique sur plusieurs gisements. Ainsi, des os du métapode portant des traces d'extraction de baguettes pour la fabrication d'aiguilles sont identifiés à travers toute l'Europe : Gönnersdorf, Petersfels, Pekarna, Solutré, au Grand Pastou (StordeurYedid, 1979; Berke, 1987; Valoch, 1996; Turner, 2002; Chauvière, 2006), rappelant ainsi l'objet identifié à Faustin. L'utilisation des os de chevaux comme support de gravure est encore plus rare. À Faustin, au moins un os a servi de support pour les figures féminines de type Gönnerdorf (Lenoir, 1983; Delporte, 1993). Cet usage des os de chevaux est aussi observé à Petersfels (Berke, 1987), Laugerie-Basse (Paillet, 2014) et Pekarna (Lázničková-Galetová, 2002).

In fine, à l'échelle de l'Europe occidentale, depuis l'Entre-deux-Mers jusqu'en Rhénanie centrale, en passant par le Bassin parisien et le plateau Suisse une certaine homogénéité dans les modes d'exploitation du cheval au Magdalénien supérieur se dessine.

Notre étude, bien que replacée dans son cadre régional, ne porte que sur un gisement. L'étude archéozoologique de gisements contemporains, tel que La Faurélie par exemple, serait sans nul doute d'un grand intérêt. Ainsi serait-il possible, à l'instar de ce qui a été réalisé pour le Bassin parisien (e. g. Audouze, 2006; Olive et Valentin, 2006; Bignon, 2008) de modéliser un des volets de l'organisation socio-économique de la fin du Magdalénien.

Remerciements : Cette étude a été menée dans le cadre d'un mémoire de master 2 à l'université de Bordeaux. Elle a pu être réalisée grâce à la gratification du projet « Magdatis » (ANR 2011 BSH3 005). Nous tenons ainsi à remercier les responsables du projet, V. Laroulandie (PACEA, université de Bordeaux) et J.-M. Pétillon (TRACES, université Toulouse - Jean-Jaurès). Nos remerciements s'adressent aussi à $\mathrm{M}$. Lenoir pour nous avoir confié la collection ainsi qu'à D. Armand (PACEA) pour avoir facilité l'accès au matériel. Merci également à C. BarshaySzmidt (University of Cambridge) pour avoir porté à notre connaissance les nouvelles dates et au laboratoire PACEA pour le financement de celles-ci. Enfin, nous tenons à remercier M. Langlais (PACEA) et V. Laroulandie pour leurs conseils et relectures.

\section{NOTES}

(1) L'individu D2 est représenté par des dents définitives qui ne sont pas sorties des alvéoles dentaires.

(2) Les dents jugales permanentes des individus $\mathrm{C} 3$ et $\mathrm{C} 4$ ne sont sorties des alvéoles dentaires.

(3) Notons toutefois l'apport de pièces de silex « grain-demil » d'origine charentaise (Langlais, comm. pers.).

\section{RÉFÉRENCES BIBLIOGRAPHIQUES}

Audouze F. (2006) - Essai de modélisation du cycle annuel de nomadisation des Magdaléniens du Bassin parisien, in $\mathrm{M}$. Olive et B. Valentin (dir.), Variabilité des habitats tardiglaciaires dans le Bassin parisien et ses alentours : quelles significations, actes de la table ronde, séance de la Société préhistorique française (Paris, 23 novembre 2005), Bulletin de la Société préhistorique française, 104, 4, p. 683-694.

BARONE R. (1986) - Anatomie comparée des mammifères domestiques, 1. Ostéologie, Paris, Vigot, 761 p.

Berke H. (1987) - Die Grossäuugerreste aus den spätpleistozänen und holozäne Horizonten der Grabungen am „Felsställe” bei Ehingen-Mühlen, Alb-Donau Kreis, in C. J. Kind, Das Felsställe. Eine jungpaläolithisch-frühmesolithisch AbriStation bei Ehringen-Mühlen, Alb-Danau Kreis. Die Grabungen 1975-1980, Stuttgart, Konrad Theiss, p. 303-338.

Berke H. (1992) - Solutré, Peterfels, Pekarna, Kniegrotte : l'homme et les chevaux magdaléniens, la chasse la boucherie et l'environnement, in J.-P. Rigaud, H. Laville et B. Vandermeersch (dir.), Le peuplement magdalénien : paléogéographie physique et humaine, actes du colloque (Chancelade, 10-15 octobre 1988), Paris, CTHS, p. 273-1276. 
Bertran P., Beauval C., Boulogne S., Brenet M., Costamagno S., Feuillet T., Laroulandie V., Lenoble A., Malaurent P., Mallye J.-B. (2015) - Experimental archaeology in a mid-latitude periglacial context: insight into site formation and taphonomic processes, Journal of Archaeological Science, 57, p. 283-301.

Bignon O. (2003) - Diversité et exploitation des équidés au Tardiglaciaire en Europe occidentale : implications pour les stratégies de subsistance et les modes de vie au Magdalénien et à l'Azilien ancien du Bassin parisien, thèse de doctorat, université Paris X, Nanterre, 856 p.

Bignon O. (2006) - Approche morphométrique des dents jugales déciduales d'Equus caballus arcelini (sensu lato, Guadelli, 1991) : critères de détermination et estimation de l'âge d'abattage, Comptes rendus de l'Académie des sciences, Palevol, 5, 8, p. 1005-1020.

Bignon O. (2008) - Chasser les chevaux à la fin du Paléolithique dans le Bassin parisien : stratégie cynégétique et mode de vie au Magdalénien et à l'Azilien ancien, Oxford, Archaeopress (BAR, International series 1747), 170 p.

Binford L. R. (1978) - Nunamiut Ethnoarchaeology, San Francisco, Academic Press (Studies in Archaeology), 509 p.

Binford L. R. (1981) - Bones: Ancient Men and Modern Myths, New York, Academic press (Studies in Archaeology), 320 p.

Blumenschine R. J., Madrigal T. C. (1993) - Variability in Long Bone Marrow Yields of East African Ungulates and its Zooarchaeological Implications, Journal of Archaeological Science, 20, p. 555-587.

Bordes F., Sonneville-Bordes D. de (1979) - L'azilianisation dans la vallée de la Dordogne : les données de la gare de Couze (Dordogne) et de l'abri Morin (Gironde), in D. de Sonneville-Bordes (dir.), La fin des temps glaciaires en Europe : chronostratigraphie et écologie des cultures du Paléolithique final, actes du colloque international du CNRS n 271 (Talence, 24-28 mai 1977), Paris, CNRS, p. $449-460$.

Boyle K. V. (1994) - La Madeleine (Tursac, Dordogne) : une étude paléo-économique du Paléolithique supérieur, Paléo, 6, p. 55-77.

Brain C. K. (1969) - The Contribution of Namib Desert Hottentots to an Understanding of Australopithecine Bone Accumulations, Scientific Papers of the Namib Desert Research Station, 39, p. 13-22.

Bridault A., Chaix L. (2009) - Réflexions sur la recomposition des spectres fauniques dans le massif Jurassien et les Alpes françaises du Nord durant le Tardiglaciaire, in G. Pion et L. Mevel (coord.), La fin du Paléolithique supérieur dans les Alpes du Nord françaises et le Jura méridional. Approches culturelles et environnementales, Paris, Société préhistorique française (Mémoires, 50), p. 59-71.

Bridault A., Bemilli C. (1999) - La chasse et le traitement des animaux, in M. Julien et J.-L. Rieu (dir.), Occupations du Paléolithique supérieur dans le Sud-Est du Bassin parisien, Paris, Maison des sciences de l'homme (DAF, 78), p. $48-64$.

Bronk Ramsey C., Scott M., Van der Plicht H. (2013) Calibration for Archaeological and Environmental Terres- trial Samples in the Time Range 26-50 ka cal. BP, Radiocarbon, 55, 4, p. 2021-2027.

Bunn H. T. (1982) - Meat-Eating and Human Evolution: Studies on the Diet and Subsistence Practices of Plio-Pleistocene of East Africa, $\mathrm{PhD}$ Thesis, University of California, Berkeley.

Chauvière F.-X. (2006) - L'animal, ressource technique pour les Magdaléniens de la falaise Pastou, in M. Dachary (dir.), Les Magdaléniens à Duruthy: qui étaient-ils? Comment vivaient-ils?, Mont-de-Marsan, conseil général des Landes, p. 19-29.

CochaRd D. (2004) - Les Léporidés dans la subsistance paléolithique du Sud de la France, thèse de doctorat, université Bordeaux 1, $354 \mathrm{p}$.

Cochard D., Brugal J.-P. (2004) - Importance des fonctions des sites dans les accumulations paléolithiques de léporidés, in J.-P. Brugal et J. Desse (dir.), Petits animaux et sociétés humaines : du complément alimentaire aux ressources utilitaires, actes des XXIV ${ }^{e}$ Rencontres internationales d'archéologie et d'histoire (Antibes, 23-25 octobre, 2003), Antibes, APDCA, p. 283-296.

Costamagno S. (1999) - Stratégies de chasse et fonction des sites au Magdalénien dans le Sud de la France, thèse de doctorat, université Bordeaux 1, $495 \mathrm{p}$.

Costamagno S. (2003) - L'exploitation des Ongulés au Magdalénien dans le Sud de la France, in S. Costamagno et V. Laroulandie (dir.), Mode de vie au Magdalénien : apports de l'archéozoologie, actes du XIVe Congrès de l'UISPP, colloque-symposium 6.4 (Liège, 2-8 septembre 2001), Oxford, Archaeopress (BAR, International series 1144), p. 73-88.

Costamagno S. (2005) - Mobilité, territoires de chasse et ressources animales au Magdalénien final en contexte pyrénéen : le niveau 7A de la grotte abri du Moulin (Troubat, Hautes-Pyrénes), in J. Jaubert et M. Barbaza (dir.), Territoires, déplacements, mobilité, échanges durant la Préhistoire. Terres et hommes $d u$ Sud, actes du $126^{\circ}$ Congrès national des sociétés historiques et scientifiques, section de Pré- et Protohistoire (Toulouse, 2001), Paris, CTHS, p. 371-383.

Costamagno S. (2006) - Archéozoologie des grands mammifères des gisements de la falaise du Pastou, in M. Dachary (dir.), Les Magdaléniens à Duruthy : qui étaient-ils? Comment vivaient-ils? Mont-de-Marsan, conseil général des Landes, p. 19-29.

Costamagno S., David F. (2009) - Comparaison des pratiques bouchères et culinaires de différents groupes sibériens vivant de la renniculture, Archeofauna, 18, p. 9-25.

Costamagno S., Laroulandie V. (2004) - L'exploitation des petits vertébrés dans les Pyrénées françaises du Paléolithique au Mésolithique : un inventaire taphonomique et archéozoologique, in J.-P. Brugal et J. Desse (dir.), Petits animaux et sociétés humaines : du complément alimentaire aux ressources utilitaires, actes des XXIV ${ }^{e}$ Rencontres internationales d'archéologie et d'histoire (Antibes, 23-25 octobre, 2003), Antibes, APDCA, p. 403-416.

Costamagno S., Cochard D., Ferrié J.-G., Laroulandie V., Cazals N., Valdeyron N., Cachary M., Barbaza M., Galop D., Martin H., Philibert S. (2008) - Nouveaux milieux, nouveaux gibiers, nouveaux chasseurs : évolution des pratiques cynégétiques dans les Pyrénées du Tardigla- 
ciaire au début du Postglaciaire, Bulletin de la Société préhistorique française, 105, 1, p. 17-27.

DelPech F. (1967) - Recherches paléontologiques concernant quelques gisements du Magdalénien VI : stations de la gare de Couze (Dordogne), du Morin (Gironde) et de Duruthy (Landes), thèse de doctorat, université Bordeaux 1, $202 \mathrm{p}$.

Delpech F. (1971) - L'abri Faustin, commune de Cessac (Gironde) : étude paléontologique, Bulletin de la Société préhistorique française, 68, 1, p. 328-332.

Delpech F. (1975) - Les faunes du Paléolithique supérieur dans le Sud-Ouest de la France, thèse de doctorat, université Bordeaux 1, $374 \mathrm{p}$.

Delpech F. (1999) - Biomasse d'ongulés au Paléolithique et inférences sur la démographie, Paléo, 11, p. 19-42.

Delpech F., Villa P. (1993) - Activités de chasse et de boucherie dans la grotte des Églises, in J. Desse et F. AudoinRouzeau (dir.), Exploitation des animaux sauvages à travers le temps, actes des $\mathrm{XIII}^{\mathrm{e}}$ Rencontres internationales d'archéologie et d'histoire (Antibes, 15-17 octobre 1992), Antibes, APDCA, p. 81-102.

Delporte H. (1993) - L'Image de la femme dans l'art préhistorique, Paris, Picard, $287 \mathrm{p}$.

Demars P.-Y. (2002) - Changements climatiques et occupation de l'espace. Les derniers chasseurs cueilleurs face à la déglaciation, Quaternaire, 13, p. 289-296.

Discamps E. (2011) - Hommes et hyènes face aux recompositions des communautés d'ongulés (MIS 5-3) : éléments pour un cadre paléoécologique des sociétés du Paléolithique moyen et supérieur ancien d'Europe de l'Ouest, thèse de doctorat, université Bordeaux 1, $437 \mathrm{p}$.

Enloe J. G. (2000) - Le Magdalénien du Bassin parisien au Tardiglaciaire : la chasse aux rennes comparée à celle d'autres espèces, in G. Pion (dir.), Le Paléolithique supérieur récent : nouvelles données sur le peuplement et l'environnement, actes de la table de ronde (Chambéry, 12-13 mars 1999), Paris, Société préhistorique française (Mémoires, 28), p. 39-45

Enloe J. G., David F. (1997) - Rangifer Herb Behavior: Seasonality of Hunting in the Magdalenian of the Paris Basin, in L. J. Jackson et P. T. Thacker, Caribou and Reindeer Hunters of the Northern Hemisphere, Aldershot (RU) et Brookfield (Vt.), Avebury, p. 55-68.

Fernandez P., Legendre S. (2003) - Mortality Curves for Horses from the Middle Palaeolithic site of Bau de l'Aubesier (Vaucluse, France): Methodological, Palaeoethnological, and Palaeo-ecological Approaches, Journal of Archaeological Science, 30, 12, p. 1577-1598.

Fisher J. W. (1995) - Bone Surface Modifications in Zooarchaeology, Journal of Archaeological Method and Theory, 2, p. 7-68.

Fontana L. (1998) - Mobilité et subsistance au Magdalénien dans le Languedoc occidental et le Roussillon, thèse de doctorat, université Paris I - Panthéon-Sorbonne, $287 \mathrm{p}$.

Fontana L. (1999) - Mobilité et subsistance au Magdalénien dans le bassin de l'Aude, Bulletin de la Société préhistorique française, 96, 2, p. 175-190.
Groves C. (1974) - Horses, Asses and Zebras in the Wild, Newton Abbot et Londres, David \& Charles, 192 p.

Kuntz D. (2006) - Données nouvelles sur les stratégies d'acquisition et de traitement des carcasses au cours du Magdalénien supérieur dans la vallée de l'Aveyron : l'exemple de Magdeleine la Plaine (Penne, Tarn), Préhistoire du Sudouest, 13, 2, p. 151-165.

Kuntz D., Costamagno S. (2011) - Relationship Between Reindeer and Man in Southwestern France During the Magdalenian, Quaternary International, 238, p. 12-24.

Lam Y. M., Chen X., Marean C. W., Frey C. J. (1998) - Bone Density and Long Bone Representation in Archaeological Faunas: Comparing Results from CT and Photon Densitometry, Journal of Archaeological Science, 25, p. 559-570.

Lam Y. M., Pearson O. M., Marean C. W., Chen X. (2003) Bone Density Studies in Zooarchaeology, Journal of Archaeological Science, 30, p. 1701-1708.

Langlais M., Costamagno S., Laroulandie V., Pétillon J.-M., Discamps E, Mallye J.-M., Cochard D., Kuntz D. (2012) - The Evolution of Magdalenian Societies in South-West France Between 18,000 and 14,000 cal. BP : changing environments changing tool kits, Quaternary international, 272-273, p. 138-149.

Langlais M., Laroulandie V., Pétillon J.-M., Mallye J.-B., Costamagno S. (2014) - Évolution des sociétés magdaléniennes dans le Sud-Ouest de la France entre 18500 et 14000 cal. BP, in J. Jaubert, N. Froment et P. Depaepe, Transitions, ruptures et continuité en Préhistoire, actes du XXVII ${ }^{e}$ Congrès préhistorique de France (Bordeaux, 2010), Paris, Société préhistorique française, p. 417-430.

LARoulandie V. (2003) - Exploitation des oiseaux au Magdalénien en France : état des lieux, in S. Costamagno et V. Laroulandie (dir.), Mode de vie au Magdalénien : apports de l'archéozoologie, actes du XIV Congrès de l'UISPP, colloque-symposium 6.4 (Liège, 2-8 septembre 2001), Oxford, Archaeopress (BAR, International series 1144), p.129-138.

Laroulandie V. (2004) - Exploitation des ressources aviaires durant le Paléolithique en France : bilan critique et perspectives, in J.-P. Brugal et J. Desse (dir.), Petits animaux et sociétés humaines : du complément alimentaire aux ressources utilitaires, actes des XXIV Rencontres internationales d'archéologie et d'histoire (Antibes, 23-25 octobre, 2003), Antibes, APDCA, p. 403-416.

LARoulandie V. (2009) - De la plume à l'œuf : exploitation des ressources aviaires au Magdalénien dans le Sud de la France, in L. Fontana, F.-X. Chauvière et A. Bridault, In search of Total Animal Exploitation. Cases Studies in Upper Palaeolithic and Mesolithic, actes du XV Congrès de l'UISPP (Lisbonne, 4-9 septembre 2006), Oxford Archaeopress (BAR, International series 2040), p. 71-89.

Laroulandie V., Mallye J.-B., Costamagno S. (2014) Diversité des ressources animales au Magdalénien classique dans le Sud-Ouest de la France, in Grands sites d'art magdalénien : la Madeleine et Laugerie-Basse il y a 15000 ans, Paris, Réunion des musées nationaux, Grand Palais, p. 42-44.

LÁZNIČKOVÁ-GALETOVÁ M. (2002) - Art mobilier magdalénien en matières dures animales de Moravie (République 
tchèque). Aspects technologique et stylistique, L'Anthropologie, 106, p. 525-264.

Le BAIL A. (2005) - L'exploitation de la chouette harfang (Nyctea scandiaca) au Magdalénien récent : étude des gisements du Faustin et de Gare de Couze, mémoire de master 2, université Bordeaux 1, $72 \mathrm{p}$.

Le Gall O. (2003) - Des Magdaléniens et... des poissons, in S. Costamagno et V. Laroulandie (dir.), Mode de vie au Magdalénien : apports de l'archéozoologie, actes du XIVe Congrès de 1'UISPP (Liège, 2-8 septembre 2001), Oxford Archaeopress (BAR, International series 1144), p. 119-128.

Lenoir M. (1983) - Le Paléolithique des basses vallées de la Dordogne et de la Garonne, thèse de doctorat, université Bordeaux 1,702 p.

Lenoir M., Terraza S. (1971) - Le Magdalénien supérieur de l'abri Faustin, commune de Cessac (Gironde), Bulletin de la Société préhistorique française, 68, p. 311-327.

Levine M. A. (1983) - Mortality Models and the Interpretation of the Population Horse Structure, in G. N. Bailey, Huntergatherers economy in Prehistory, Cambridge, Cambridge University Press, p. 22-46.

LuPo K. D. (1998) - Experimentally Derived Extraction Rates for Marrow: Implications for Body Part Exploitation Strategies of Plio-Pleistocene Hominid Scavengers, Journal of Archaeological Science, 25, p. 657-675.

Lupo K. D., Schmidt D. N. (1997) - Experiments in Bone Boiling: Nutritional Returns and Archaeological Reflections, Anthropozoologica, 25-26, p. 137-144.

LYMAN R. L. (1994a) - Quantitative Units and Terminology in Zooarchaeology, American Antiquity, 25, p. 36-71.

Lyman R. L. (1994b) - Vertebrate Taphonomy, Cambridge, Cambridge University Press (Cambridge Manuals in Archaeology), $524 \mathrm{p}$.

Mallye J.-B. (2007) - Les restes de blaireau en contexte archéologique : taphonomie, archéozoologie et éléments de discussion des séquences préhistoriques, thèse de doctorat, université Bordeaux 1, 548 p.

Mallye J.-B., Costamagno S., Laroulandie V., Beauval C. (2009) - Impacts des processus périglaciaires sur la préservation des ossements, Nouvelles de l'Archéologie, 118 (Programmes expérimentaux en taphonomie), p. 26-31.

Mallye J.-B., Kuntz D., Langlais M., BoudadiMaligne M., Barshay-Szmidt C., Costamagno S., Pétillon J.-M., Gourichon L., Laroulandie V. (en préparation) - Trente ans après, que reste-t-il du modèle d'azilianisation proposé au Morin par F. Bordes et D. de Sonneville-Bordes?

Moigne A.-M. (2003) - Les faunes de grands mammifères de la grotte des Conques à Vingrau, in $\mathrm{H}$. Baills (dir.), Les Conques. Des chasseurs et leur territoire, Liège, université de Liège, service de Préhistoire (ERAUL, 101), p. 75-103.

Morel P., Müller W. (1997) - Hauterive-Champréveyres, 11. Un campement magdalénien au bord du lac de Neuchâtel : étude archéozoologique (secteur 1), Neuchâtel, office du Patrimoine et de l'Archéologie (Archéologie neuchâteloise, 23), $149 \mathrm{p}$.
MüLleR W. (2006) - Les témoins animaux, in J. Bullinger, D. Leesch et N. Plumettaz (dir.), Le site magdalénien de Monruz, 1. Premiers éléments pour l'analyse d'un habitat de plein air, Neuchâtel, office du Patrimoine et de l'Archéologie (Archéologie neuchâteloise, 33), p. 123-137.

MüLLER W. (2014) - Le site magdalénien de Monruz, 3. Acquisition, traitement et consommation des ressources animales, Neuchâtel, office du Patrimoine et de l'Archéologie (Archéologie neuchâteloise, 49), 309 p.

NiLlsen P. J. (2000) - An Actualistic Butchery Study in South Africa and its Implications for Reconstructing Hominid Strategies of Carcass Acquisition and Butchery in the Upper Pleistocene and Plio-Pleistocene, PhD Thesis, University of Cape Town, Le Cap, 649 p.

Olive M., Valentin B. (2006) - Variabilité des habitats tardiglaciaires dans le Bassin parisien et ses alentours : quelles significations?, actes de la table ronde, séance de la Société préhistorique française (Paris, 23 novembre 2005), Bulletin de la Société préhistorique française, 106, 4, p. 667-790.

Olsen S. L. (1989) - Solutré: a Theoretical Approach to the Reconstruction of Upper Paleolithic Hunting Strategies, Journal of Human Evolution, 18, p. 295-327.

Outram A. K., Rowley Conwy P. (1998) - Meat and Marrow Utility Indices for Horse (Equus), Journal of Archaeological Science, 25, 9, p. 839-849.

Pailhaugue N. (1995) - La faune de la salle Monique, grotte de la Vache (Alliat, Ariège), Bulletin de la société préhistorique de l'Ariège, 50, p. 225-289.

Pailhaugue N. (1998) - Faune et saisons d'occupation de la salle Monique au Magdalénien pyrénéen, grotte de la Vache (Alliat, Ariège, France), Quaternaire, 9, p. 385-400.

Paillet P. (2014) - L'art des objets de la Préhistoire. LaugerieBasse et la collection du marquis Paul de Vibraye au Muséum national d'histoire naturelle, Arles, Errance, 175 p.

Poplin F. (1992) - Les restes osseux animaux de l'habitat magdalénien de Marsangy, in B. Schmider (dir.), Marsangy, un campement des derniers chasseurs magdaléniens sur les bords de l'Yonne, Lièges, université de Liège, service de Préhistoire (ERAUL, 55), p. 37-44.

Poplin F. (1994) - La faune d’Étiolles : milieu animal, milieu taphonomique, milieu humain, in Y. Taborin (dir.), Environnements et habitats magdaléniens dans le centre du Bassin parisien, Paris, Maison des sciences de l'homme (DAF, 43), p. 94-104.

Prummel W. (1987) - Altas for Identification of Fœtal Skeletal Elements of Cattle, Horse, Sheep and Pig, Archaeozoologica, 1987, 1, p. 23-30.

Prummel W. (1988) - Altas for Identification of Fœtal Skeletal Elements of Cattle, Horse, Sheep and Pig, Archaeozoologica, 1988, 1, p. 11-52.

Prummel W. (1989) - Altas for Identification of Fœtal Skeletal Elements of Cattle, Horse, Sheep and Pig, Archaeozoologica, 1989, 1, p. 13-26.

Rasmussen S. O., Bigler M., Blockley S. P., Blunier T., Buchardt S. L., Clausen H. B., Cvijanovic I., DahlJensen D., Johnsen S. J ., Fischer H., Gkinis V., GuilLevic M., Hoek W. Z., Lowe J. J., Pedro J. B., Popp T., 
Seierstad I. K., Steffensen J. P., Svensson A. M., ValLelonga P., Vinther B. M., Walker M. J., Wheatley J. J., WinstruP M. (2014) - A Stratigraphic Framework for Abrupt Climatic Changes during the Last Glacial Period Based on Three Synchronized Greenland Ice-core Records: Refining and Extending the INTIMATE Event Stratigraphy, Quaternary Science Review, 106, p. 14-28.

Soulier M.-C., Kuntz D., Lacarrière, Castel J.-C. (2014) - Le renne comme ressource alimentaire : discussion entre pratiques actuelles et paléolithiques, in $\mathrm{S}$. Costamagno (dir.), Histoire de l'alimentation humaine : entre choix et contraintes, actes du $138^{\mathrm{e}}$ Congrès national des sociétés historiques et scientifiques (Rennes, 2013), Paris, CTHS, p. 153-169.

Stanford J. D., Rohling E. J., Bacon S., Roberts A. P., Grousset F. E., Bolshawa M. (2011) - A New Concept for the Paleo-oceanographic Evolution of Heinrich Event 1 in the North Atlantic, Quaternary Science Review, 30, p. 1047-1066.

Stordeur-Yedid D. (1979) - Les aiguilles à chas au Paléolithique, Paris, CNRS, $215 \mathrm{p}$.

Street M. (1997) - Aspects of Late Upper Palaeolithic Settlement and Chronology in Northern Central Europe, in B. Valentin, P. Bodu et M. Christensen (dir.), L'Europe central et septentrionale au Tardiglaciaire : confrontation des modèles régionaux de peuplement, actes de la table ronde internationale (Nemours, 14-16 mai 1997), Nemours, musée de Préhistoire d'île-de-France (Mémoires, 7), p. 55-71.

Street M., Gelhaussen F., Grimm S., Moseler F., Niven L., Sensburg M., Turner E., Wenzel S., Jöris O. (2006) L'occupation du basin de Neuwied (Rhénanie central, Allemagne) par les Magdaléniens et les groupes à Ferdermesser (Aziliens), Bulletin de la Société préhistorique française, 103, 4, p. 753-780.

Street M., Jöris O., Turner E. (2012) - Magdalenian Settlement in the German Rhineland. An Update, Quaternary International, 272-273, p. 231-250.
Street M., Turner E. (2013) - The Faunal Remains from Gönnersdorf, Mayence, Römisch-Germanischen Zentralmuseums, $357 \mathrm{p}$.

Turner E. (2002) - Solutré: an Archeozoological Analysis of the Magdalenian Horizon, Mayence, Römischgermanisches Zentralmuseum (Monographie, 46), 166 p.

VAL A. (2009) - Le dépouillement des petits carnivores par les taxidermistes et ses conséquences sur le squelette : implications pour le Paléolithique en Europe, mémoire de master 2, université Bordeaux 1,72 p.

Valoch K. (1996) - Le Paléolithique en Tchéquie et en Slovaquie, Grenoble, Jérôme Millon, p. 358.

Villa P., Mahieu E. (1991) - Breakage Patterns of Human Long Bones, Journal of Human Evolution, 21, p. 27-48.

Weniger G. C. (1987) - Magdalenian Settlement and Subsistence in South-west Germany, Proceedings of the Prehistoric Society, 53, p. 293-307.

West D. (1997) - Hunting Strategies in Central Europe during the Last Glacial Maximum, Oxford, Archaeopress (BAR, International Series 672), $153 \mathrm{p}$.

Léa FEyfant

David COCHARD

Jean-Baptiste MALLYE

UMR 5199 « PACEA»

université de Bordeaux

Allée Geoffroy Saint-Hilaire CS 5002333615 PESSAC Cedex

lea.feyf@gmail.com david.cochard@u-bordeaux.fr jb.mallye@pacea.u-bordeaux1.fr 